\title{
Electronic shells or molecular orbitals: Photoelectron spectra of $\mathrm{Ag}_{n}^{-}$ clusters
}

\author{
H. Handschuh, Chia-Yen Cha, P. S. Bechthold, G. Ganteför, and W. Eberhardt \\ Institut für Festkörperforschung, Forschungszentrum Jülich, 52425 Jülich, Germany
}

\begin{abstract}
Photoelectron spectra of $\mathrm{Ag}_{n}^{-}$clusters with $n=1-21$ recorded at different photon energies $(h \nu=4.025,4.66,5.0$, and $6.424 \mathrm{eV})$ are presented. Various features in the spectra of $\mathrm{Ag}_{2}^{-}-\mathrm{Ag}_{9}^{-}$can be assigned to electronic transitions predicted from quantum chemical ab initio calculations. While this comparison with the quantum chemical calculations yields a detailed and quantitative understanding of the electronic structure of each individual cluster, a discussion in terms of the shell model is able to explain trends and dominant patterns in the entire series of spectra up to $\mathrm{Ag}_{21}^{-}$.
\end{abstract}

\section{INTRODUCTION}

One of the most important concepts of physics is that of fermions trapped in a potential with spherical symmetry. It can be used to explain the Periodic Table of the elements as well as the structure of the atomic nuclei. Recently it was also applied to a new field: the electronic structure of clusters of simple metals. ${ }^{1}$ Small particles containing up to several hundred alkali metal atoms exhibit outstanding features in the size dependence of their properties like ionization potentials or stabilities. These lead to the so-called magic numbers which are reminiscent of the variations of, e.g., the ionization potentials of the atoms or the outstanding stability of certain nuclei. Accordingly, an electronic shell model has been developed for the valence electrons in alkali metal clusters. ${ }^{1}$ The clusters are roughly described as metal spheres wherein the $s$ electrons are delocalized. This corresponds to the quantum mechanical problem of fermions confined in a spherical potential. The solutions are eigenstates with a defined angular momentum and a certain degree of degeneracy.

In principle, these shells can be directly observed using photoelectron spectroscopy. However, several experimental difficulties have to be solved. The clusters have to be mass separated, the internal temperature of the clusters has to be kept as low as possible, and the energy resolution of the spectrometer should be sufficient to resolve the electronic fine structure. The problem of mass separation is easily solved by the technique of photoelectron spectroscopy of negatively charged particles. ${ }^{2-9}$ However, in earlier experiments on alkali ${ }^{6}$ and coinage $(\mathrm{Cu}, \mathrm{Ag}, \mathrm{Au})$ metal cluster anions $^{2,10-13}$ either the spectral resolution ${ }^{3,411-13}$ or the spectral range $e^{2,6,10}$ (= photon energy) were limited and the details of the electronic structure of these particles could not be revealed.

We recently set up a new experiment ${ }^{9}$ using either a laser vaporization source ${ }^{14}$ or a pulsed arc cluster ion source $(\mathrm{PACIS})^{15}$ for the generation of cold (dimer vibrational temperature $<200 \mathrm{~K})^{16,17}$ anions and an electron spectrometer with an energy resolution of $8-30 \mathrm{meV}^{17} \mathrm{In}$ an earlier paper ${ }^{18}$ we published first data on $\mathrm{Cu}$ cluster anions, which were compared to predictions of the shell model.

In the present contribution we focus on Ag clusters. We consider Ag as a model material for probing the shell model.
Figure 1 displays a schematic comparison of the density of states of the valence bands ${ }^{19}$ of bulk $\mathrm{Na}$ with the coinage metals. The bandwidth of the occupied states of $\mathrm{Cu}$ and $\mathrm{Ag}$ is about two times larger than the bandwidth of an alkali metal. In $\mathrm{Cu}$ the top of the $d$ density of states is located $2 \mathrm{eV}$ below the Fermi energy $\left(E_{F}\right)$ while it is at about $4 \mathrm{eV}$ in the case of $\mathrm{Ag}$. From this schematic view a similarity of the electronic structure of the uppermost valence orbitals of $\mathrm{Cu}$ and $\mathrm{Ag}$ clusters can be expected. Within $4 \mathrm{eV}$ below the highest occupied molecular orbital (HOMO) the electronic structure of Ag clusters should be similar to the structure of alkali metal clusters except for a scaling factor that accounts for the difference in bandwidth. For Au the $5 d$ density of states is split into two components partially due to the large spinorbit interaction.

As will be demonstrated below, the observed spectral patterns of Ag clusters are much sharper than the corresponding data on $\mathrm{K}$ or Na clusters. ${ }^{6}$ The reason for this might be the more rigid structure of the Ag clusters at a given temperature due to the stronger bonding. Therefore, the fine structure in the Ag data presented in this paper may also serve to clarify the picture of the electronic structure of clusters of simple metals.

\section{EXPERIMENTAL SETUP}

The experimental setup is described in detail elsewhere. ${ }^{9}$ For the generation of $\mathrm{Ag}_{n}^{-}$clusters a laser vaporization source $^{14}$ is used. A Ag rod is hit by a high energy laser pulse (KrF excimer laser, $h \nu=5.0 \mathrm{eV}$, ca. $0.1 \mathrm{~J} /$ pulse). The metal plasma is cooled by a He carrier gas and flushed through a 10 $\mathrm{mm}$ long channel (3 $\mathrm{mm}$ diameter) into the vacuum. The resulting supersonically expanded cluster beam contains a considerable amount of negatively charged clusters. The internal temperature of these anions can be estimated from the vibrational temperature of the dimer. For $\mathrm{Au}_{2}^{-}$a temperature of about $200 \mathrm{~K}$ has been determined. ${ }^{16,17}$ The temperature depends critically on the parameters of the supersonic expansion.

After passing a skimmer the anions are accelerated in a pulsed electric field. The anion beam is directed into the source region of an electron spectrometer. According to the differences in velocity the anion beam separates into a chain 


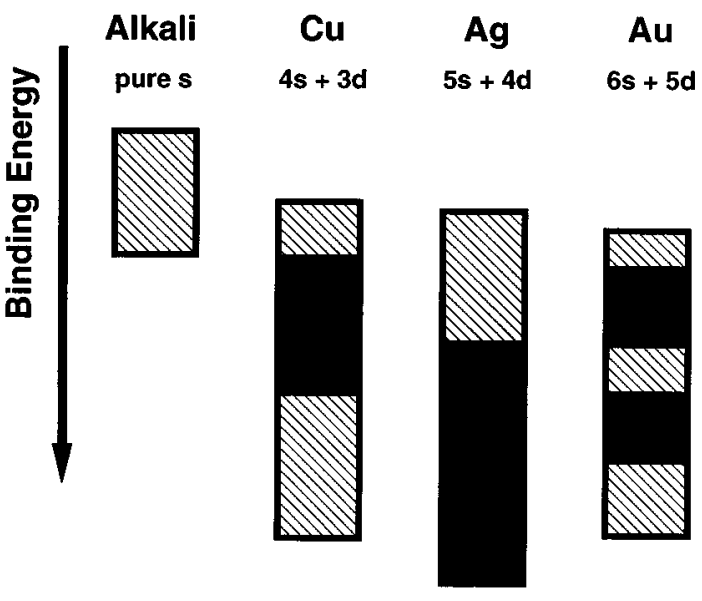

FIG. 1. Schematic comparison of the bulk density of states (Ref. 19) of an alkali metal with $\mathrm{Cu}, \mathrm{Ag}$, and $\mathrm{Au}$. The binding energy scale refers to the vacuum level. The range of binding energies covered by $s$-derived states is marked by a dashed rectangle. The $d$ partial density of states is marked by a solid black rectangle. In the case of bulk Au the $5 d$ density of states exhibits two maxima corresponding to the two spin orbit components.

of bunches of clusters with different masses (time-of-flight mass spectrometer). A selected bunch is decelerated in the center of the electron spectrometer and subsequently irradiated by an uv-laser pulse. The kinetic energy of the detached electrons is measured using a "magnetic bottle" time-offlight electron spectrometer. ${ }^{20}$ The energy resolution depends on the kinetic energy of the electrons and the velocity of the clusters. Accordingly, a maximum resolution $(<10 \mathrm{meV})$ is achieved for slow electrons ( $<1 \mathrm{eV}$ kinetic energy) and clusters of a high mass $(>500 \mathrm{amu}){ }^{17}$ For the data presented here the resolution varies between 20-100 meV. Except for the atom and the trimer all observed linewidths of the electronic transitions are large compared to the experimental resolution.

The flux of the detachment laser is about $10 \mathrm{~mJ} / \mathrm{cm}^{2}$. At higher intensities multiphoton processes induce fast fragmentation processes which yield additional features in the spectra. Except for $\mathrm{Ag}_{4}^{-}$(see below) no evidence for fragmentation has been observed in the spectra presented here. A single run is averaged for about 10000 shots $(20 \mathrm{~Hz}=8$ $\mathrm{min}$ ). Each displayed spectrum is a sum of several runs (except for $\mathrm{Ag}_{2}^{-}$).

The binding energy scale is calibrated to the electron affinity of the atom ${ }^{21}$ and the two dominant features ${ }^{10}$ in the spectrum of $\mathrm{Ag}_{3}^{-}$. Due to fluctuations of the work function of the surfaces within the electron spectrometer the absolute $\mathrm{BE}$ scale is uncertain by about $\pm 50 \mathrm{meV}$.

\section{BASIC MODELS}

\section{A. Quantumchemical calculations}

Two different theoretical $a b$ initio frameworks are common for the structural determination of metal clusters: (i) density functional calculations in the local density approximation (LDA) ${ }^{22}$ and (ii) Hartree-Fock configuration interaction (HF-CI) derived calculations. ${ }^{23,24}$ The LDA calculations deliver accurate data on ground state properties of the anion and the neutral, but a calculation of excited states is more difficult. The LDA can be combined with simulated annealing techniques ${ }^{22}$ yielding significant information about the geometric structure of a ground state cluster. This corresponds to an unrestricted search for the global minimum of the geometrical configuration space of the cluster. Such calculations result in the prediction of the $\mathrm{BE}$ of the feature at lowest $\mathrm{BE}$ in the photoelectron spectra. This energy is the vertical detachment energy (VDE), which is related to the vertical electron affinity. ${ }^{25}$

Within the HF-CI approach ${ }^{23,24}$ electronic ground and excited states of the neutral and negatively charged cluster can be calculated. A certain electronic state of a particle is described by a linear combination of certain configurations, each configuration being described by a combination of single particle molecular wave functions of the cluster. Within this framework the geometry cannot be easily determined, since a geometry optimization like the simulated annealing technique requires in the case of larger clusters too much of computer time. However, within certain symmetry restrictions the optimum geometry can be determined. In general, this geometry does not necessarily correspond to the global minimum in the geometrical configuration space of the cluster.

The differences in energy between the anion electronic ground state and the electronic states of the neutral cluster in the geometry of the anion correspond to the positions of the peaks observed in the photoelectron spectra. These peak positions have recently been calculated for $\mathrm{Ag}_{n}^{-}$clusters with $n=2-9$ by Bonacic-Koutecky et al. ${ }^{24}$ in the HF-CI approach. The $4 d$ electrons are included using a relativistic effective core potential approximation.

\section{B. Electronic shell model}

The electronic structure of a metal cluster with highly delocalized valence electrons can be described by the electronic shell model. ${ }^{1}$ It assumes that the electrons can move freely within the boundaries of the cluster. If the geometric structure of the cluster is approximately spherical, the single particle eigenstates are electronic shells with a defined angular momentum. The clusters with a number of valence electrons corresponding to a shell closing have a high ionization potential, a low electron affinity, a low reactivity and high stability similar to a rare gas atom. Clusters with an additional electron behave analogous to an alkali atom. The shell closings occur at $n_{e}=2,8,18,20,34,40, \cdots$ electrons corresponding to the filling of the $1 s, 1 p, 1 d, 2 s, 1 f, \cdots$ shells, respectively. Analogous to the atomic nuclei only the clusters with a number of electrons corresponding to a shell closing are spherical. In between the clusters exhibit a prolate or oblate deformation. This deformation corresponds to a lifting of the degeneracy of the sublevels of the shells. On the other hand, from an observed splitting the deformation of a cluster can be determined.

The shell model neglects the influence of the core potentials of the individual atoms of the clusters. The positive charge of the atomic cores is smeared out to a homogenous background (jellium model). Therefore, an accurate description of the structure similar to the quantum chemical treat- 
ment is difficult based on the shell model. However, the shell model approximation is very useful to compare clusters within a series with increasing size and to study trends and patterns in the transition from the atom to the bulk.

\section{Photoelectron spectroscopy}

Photoelectron spectra of negatively charged particles can be interpreted using two different models. The quantum mechanical exact description assigns each feature to a transition from a vibronic state of the anion to a vibronic state of the neutral particle. Since the anions are cooled in a supersonic expansion, they should be in their electronic ground state. Only in a few cases features have been observed in the spectra which were assigned to transitions from electronically excited anions present in the beam. ${ }^{25}$ If the anion is in its electronic ground state, in most cases the feature at lowest $\mathrm{BE}$ corresponds to the transition into the electronic ground state of the neutral. The time scale of the detachment process is fast with respect to the movements of the nuclei. Therefore, the observed features correspond to the electronic states of the neutral cluster having the ground state geometry of the anion. A comparison with data on neutral clusters (e.g., with resonant two-photon ionization experiments) is only possible, if this difference in geometry is taken into account. ${ }^{26}$

On the other hand, the spectra can also be discussed within a much simpler picture: The observed features are assigned to photoemission from occupied single particle orbitals. Within this approximation a spectrum represents a picture of the electronic shell structure. However, this approach can be used for a qualitative interpretation only while the following four effects must be considered.

(i) The emission of an electron corresponds to a change of the charge of the cluster. The remaining electrons adjust to the changed potential. This fast relaxation process yields energy, which in general is transferred to the outgoing electron. Therefore, the binding energy of a single particle orbital determined from a photoelectron spectrum is usually significantly smaller than the calculated binding energy. The amount of energy gained by the relaxation is difficult to determine and it may differ for different single particle orbitals.

(ii) If the anion has an unpaired electron the photoemission from one of the doubly occupied single particle orbitals results in a neutral final state with two unpaired electrons. The two electrons can combine to singlet or triplet states with a slightly different energy. Therefore, photoemission from one single particle orbital can yield more than one feature. In general this multiplet splitting can be identified in the spectrum because it occurs for all occupied single particle orbitals of the cluster in a similar pattern and a characteristic intensity ratio equivalent to the degree of degeneracy ( singlet/triplet $=1 / 3)$.

(iii) The photoemission of one electron can induce the simultaneous excitation of other electrons. The configuration of the final state of a transition corresponding to direct photoemission differs from the configuration of the initial state by the lack of just one electron. A second electron can be excited simultaneously into a higher, unoccupied single particle orbital. If the corresponding final state has the same symmetry as the one of the direct emission process this transition is a shake-up process with respect to the direct photoemission. Usually the direct emission feature has a significant larger intensity than the shake-up peak.

It is also possible that the photoemission is accompanied by the excitation of several electrons. In addition, in some cases the symmetry of the final state of a shake-up transition is different from the symmetry of the final state of the direct emission transition (e.g., conjugated shake-up). It is not always possible to relate this transition to a direct emission process. Since we are mainly interested in the identification of the direct emission features we will label all peaks involving the excitation of more than one electron as shake-up features without a further discussion.

These shake-up features are difficult to identify in the spectrum. The relative intensity of such a feature is expected to be small with respect to direct emission features. However, the shake-up intensity depends on details of the involved wave functions and the photon energy and might be relatively high under certain conditions, especially near the ionization threshold, a condition that corresponds to our experiment.

(iv) The initial state (=electronic ground state of the anion) is described by a certain configuration, i.e., the distribution of the valence electrons into the lowest single particle orbitals of the anion. As long as the electronic states of the neutral cluster can be described by a single configuration based on the corresponding single particle orbitals of the neutral system the direct emission picture can be applied. Usually, however, the higher excited states of the neutral cluster which correspond to photoemission from single particle orbitals at higher binding energies have to be described as a linear combination of several configurations. This configuration mixing corresponds to a break down of the single particle picture. Accordingly, the picture is limited to low lying excited states corresponding to emission from the uppermost orbitals. We consider the simple picture as useful as long as the final state can be described by a leading configuration with a contribution of more than $50 \%$ to the charge density.

In an earlier publication ${ }^{18}$ we tentatively assigned certain features in the photoelectron spectra of $\mathrm{Cu}_{n}^{-}$clusters to photoemission from electronic shells based on the above considerations. Analogous assignments can be made for the $\mathrm{Ag}_{n}^{-}$ data presented here. Since the configurations and the symmetries of the single particle orbitals of at least some of the involved electronic states are known from the HF-CI calculations for $\mathrm{Ag}_{n}^{-}$, the validity of this tentative assignment can be checked.

\section{RESULTS AND DISCUSSION}

In the following we will present the photoelectron data of each $\mathrm{Ag}_{n}^{-}$cluster individually and compare the observed features to the results of the HF-CI calculations ${ }^{24}$ and the predictions of the shell model. ${ }^{1}$ In some cases the spectra are compared to the data of other simple metal clusters $(\mathrm{Cu}, \mathrm{Au}$, alkali metals) to clarify the assignments. The spectra are recorded at different photon energies to study cross section effects useful for the assignment of direct photoemission fea- 


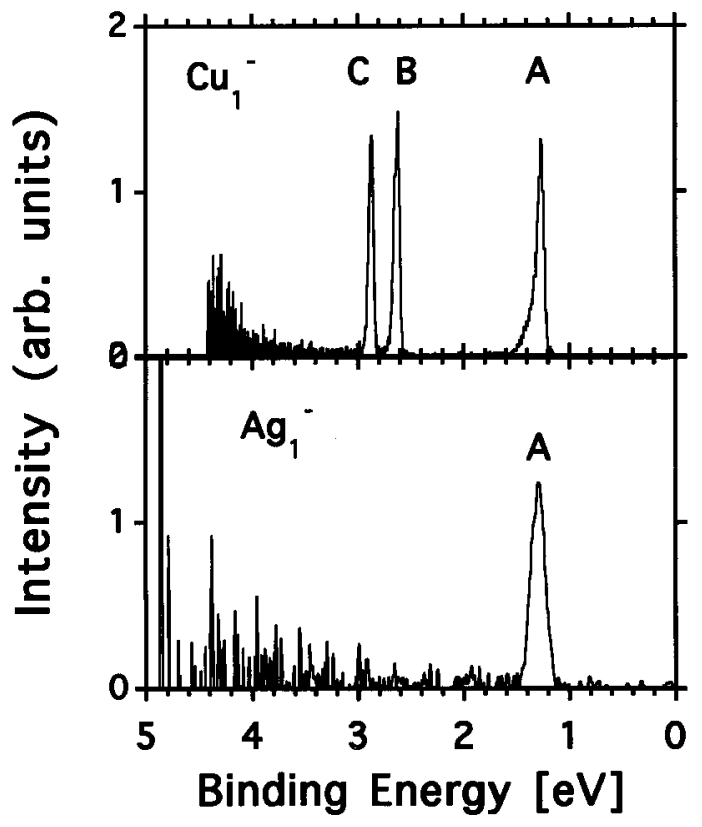

FIG. 2. A comparison of photoelectron spectra of $\mathrm{Ag}_{1}^{-}$and $\mathrm{Cu}_{1}^{-}$recorded at a photon energy of $h \nu=5.0 \mathrm{eV}$. For the assignment of the marked features $(\mathrm{A}, \mathrm{B}$, and $\mathrm{C})$ see the text.

tures and shake-up peaks. In addition, the energy resolution is best at very low kinetic energies of the electrons requiring the recording of spectra at different photon energies.

In general, the assignment of the peaks to transitions from the anion ground states into neutral electronic states is governed by a simple pattern. The anions with an even number of electrons have a singlet ground state. Then, according to the selection rules of photoemission the final states can only be doublet states. The anions with an odd number of electrons usually have a doublet ground state corresponding to an unpaired electron in the uppermost orbital. The peaks in the spectra of these anions are assigned to either singlet or triplet states. Therefore, the number of peaks is roughly twice as large in the spectra of the latter ones. With increasing cluster size the energy separation between corresponding singlet and triplet states decreases. Beyond $n=8$ this spacing is smaller than the average width of the observed peaks.

In the following the data of each cluster are discussed individually. The positions of the experimentally identified peaks are listed in Table I. The features are compared to the results of the HF-CI calculations ${ }^{24}$ for $n=2-9$. The resulting assignments and calculated BE's are also included in Table I. The dominant features are also compared to the predictions of the electronic shell model. ${ }^{1}$ The corresponding assignments are listed in Table I, too.

$A g_{1}^{-}$

Figure 2 displays a comparison of photoelectron spectra of $\mathrm{Ag}_{1}^{-}$and $\mathrm{Cu}_{1}^{-}$recorded with $h \nu=5.0 \mathrm{eV}$. The feature at the lowest $\mathrm{BE}$ (marked $\mathrm{A}$ ) corresponds to the transition into the neutral ground state. The position of the peak is equal to the electron affinity ${ }^{21}(\mathrm{Cu}: 1.226 \mathrm{eV}, \mathrm{Ag}: 1.303 \mathrm{eV})$. This is roughly twice the affinity ${ }^{21}$ of an alkali metal (Na: $0.546 \mathrm{eV}$, $\mathrm{K}: 0.501 \mathrm{eV}$ ) in agreement with the scheme illustrated in Fig. 1. The two peaks in the photoelectron spectrum of $\mathrm{Cu}_{1}^{-}$at

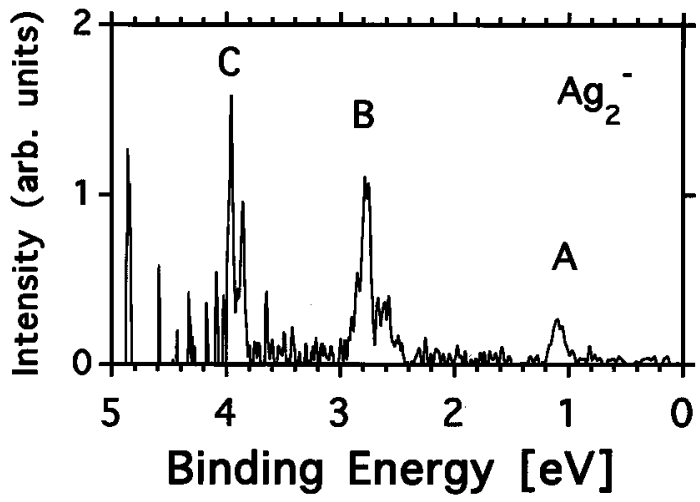

FIG. 3. Photoelectron spectrum of $\mathrm{Ag}_{2}^{-}$. The photon energy is $h \nu=5.0 \mathrm{eV}$. Marked features (A, B, and C) are discussed in the text.

about $2.8 \mathrm{eV} \mathrm{BE}$ (B and $\mathrm{C}$ ) correspond to transitions into two excited states of neutral $\mathrm{Cu}$. The configuration ${ }^{21}$ of $\mathrm{Cu}_{1}^{-}$is $3 d^{10} 4 s^{2}$, the configuration ${ }^{27}$ of the two excited states is $3 d^{9} 4 s^{2}$. Accordingly, the two peaks (B and C) can be assigned to photoemission from the $3 d$ shell of the atom. In the final state the residual nine $d$ electrons can combine to a $J=3 / 2$ and a $J=5 / 2$ state yielding two peaks due to multiplet splitting. The BE's of the peaks and the assignments are listed in Table I.

The major difference between $\mathrm{Ag}$ and $\mathrm{Cu}$ is the $\mathrm{BE}$ of the $d$-derived orbitals. In the case of $\mathrm{Ag}$ the $4 d$ shell is located at about $2 \mathrm{eV}$ higher $\mathrm{BE}$ compared to $\mathrm{Cu}$ and beyond the limit given by the photon energy. From a study of the evolution of the $3 d$ band of $\mathrm{Cu}_{n}^{-}$clusters ${ }^{11}$ it is known, that the $\mathrm{BE}$ of the $d$-derived orbitals increases monotonically. Since the $\mathrm{BE}$ of the $4 d$ orbital of the $\mathrm{Ag}$ atom is already beyond the $\mathrm{BE}$ range visible with $h \nu=5 \mathrm{eV}$ photons, we assume that the features observed in the spectra of $\mathrm{Ag}_{n}^{-}$clusters using this photon energy display only $5 s / p$-derived valence orbitals.

$\mathrm{Ag}_{2}^{-}$

Figure 3 shows the spectrum of $\mathrm{Ag}_{2}^{-}$recorded with $h \nu$ $=5.0 \mathrm{eV}$ photon energy. The relative intensity of $\mathrm{Ag}_{2}^{-}$in the mass spectrum is extremely low. Therefore, the signal to noise ratio of the photoelectron spectrum is low. Three features can be identified in the photoelectron spectrum marked A, B, and C. Other structures in the spectrum are not reproducible.

The three features A, B, and C are assigned ${ }^{24}$ to transitions from the electronic ground state of the anion ${ }^{2} \Sigma_{u}^{+}$ $\left(\sigma^{2} \sigma^{*}\right)$ to the ground state of the neutral ${ }^{1} \Sigma_{g}^{+}\left(\sigma^{2}\right)$ and the two excited states ${ }^{3} \Sigma_{u}^{+}\left(\sigma \sigma^{*}\right)$ and ${ }^{1} \Sigma_{u}^{+}\left(\sigma \sigma^{*}\right)$, respectively. The calculated peak positions are in excellent agreement with the experiment, except that the singlet/triplet splitting of the ${ }^{3} \Sigma_{u}^{+} /{ }^{1} \Sigma_{u}^{+}$states is overestimated in the calculation by about $0.3 \mathrm{eV}$. The BE's of the peaks and the assignments are listed in Table I.

In the dimer the two $4 s$ atomic orbitals combine to a bonding $\sigma$ and an antibonding $\sigma^{*}$ orbital. In the single particle picture of photoemission two peaks are expected according to the occupation of the two orbitals by the three electrons of the anion $\sigma^{2} \sigma^{*}$. However, the emission of one of the two electrons occupying the bonding $\sigma$ orbital leaves two 
TABLE I. Listing of the positions of the features found in the PES of the $\mathrm{Ag}_{n}^{-}$clusters. The peak positions are given as the peak maxima (corresponding to vertical detachment energies). The theoretical values (Ref. 26) of the features assigned to certain transitions are also included (italics) for $n=2,3,4,5,6$, and 8. Features marked isomer are assigned to photoemission from a second isomer, which is not the most abundant isomer. Features marked SU are assigned to shake-up processes (see Sec. III). Transitions into singlet or triplet states with identical configurations are marked S or T, respectively. For clusters larger than $\mathrm{Ag}_{7}^{-}$, the singlet/triplet splitting is smaller than the FWHM of the observed features. In these cases only the triplet electronic states are listed ( $\left.\mathrm{Ag}_{8}^{-}\right)$or the splitting is neglected. The electronic shell configuration for the electronic ground state is given for each anion. The configuration $1 s^{2} 1 p^{6} 1 d^{10}$ is abbreviated as $(s p d)$. The subshells are marked $p_{1}, p_{2}, p_{3}, d_{1}, d_{2}, d_{3}, d_{4}, d_{5}$. The assignment of a feature to photoemission from an electronic shell is indicated by a -1 exponent (e.g., $1 p_{1}^{-1}$ ). All values are given in $\mathrm{eV}$.

\begin{tabular}{|c|c|c|c|c|c|c|c|c|c|}
\hline \multirow[b]{2}{*}{$n$} & \multirow{2}{*}{$\begin{array}{l}\text { Anion } \\
\text { grd. state }\end{array}$} & \multirow{2}{*}{$\begin{array}{l}\text { Neutral } \\
\text { grd. state } \\
\text { A }\end{array}$} & \multicolumn{2}{|c|}{$\begin{array}{c}\text { Neutral } \\
\text { excited states } \rightarrow\end{array}$} & \multirow[b]{2}{*}{$\mathrm{D}$} & \multirow[b]{2}{*}{$\mathrm{E}$} & \multirow[b]{2}{*}{$\mathrm{F}$} & \multirow[b]{2}{*}{$\mathrm{G}$} & \multirow[b]{2}{*}{$\mathrm{H}$} \\
\hline & & & B & $\mathrm{C}$ & & & & & \\
\hline \multirow[t]{3}{*}{1} & & $1.303^{\mathrm{a}}$ & $5.053^{\mathrm{b}}$ & $5.607^{\mathrm{b}}$ & & & & & \\
\hline & ${ }^{2} S_{0}$ & ${ }^{1} S_{1 / 2}$ & ${ }^{2} D_{5 / 2}$ & ${ }^{2} D_{3 / 2}$ & & & & & \\
\hline & $1 s^{2}$ & $1 s^{-1}$ & & & & & & & \\
\hline \multirow[t]{4}{*}{2} & & 1.1 & 2.8 & 3.9 & & & & & \\
\hline & & 1.11 & 2.72 & 4.16 & & & & & \\
\hline & $1^{2} \Sigma_{u}^{+}$ & $1^{1} \Sigma_{g}^{+}$ & $1^{3} \Sigma_{u}^{+}$ & $1^{1} \Sigma_{u}^{+}$ & & & & & \\
\hline & $1 s^{2} 1 p_{1}^{1}$ & $1 p_{1}^{-1}$ & $1 s^{-1}(\mathrm{~T})$ & $1 s^{-1}(\mathrm{~S})$ & & & & & \\
\hline \multirow[t]{4}{*}{3} & & 2.43 & 3.62 & 4.83 & 5.57 & 5.80 & 6.10 & & \\
\hline & & 2.45 & 3.65 & 4.88 & 5.52 & 5.83 & & & \\
\hline & $1^{1} \Sigma_{g}^{+}$ & $1^{2} \Sigma_{u}^{+}$ & $1^{2} \Sigma_{g}^{+}$ & $2^{2} \Sigma_{g}^{+}$ & $1^{2} \Pi_{u}^{+}$ & $2^{2} \Sigma_{u}^{+}$ & & & \\
\hline & $1 s^{2} 1 p_{1}^{2}$ & $1 p_{1}^{-1}$ & $1 s^{-1^{5}}$ & $\mathrm{SU}^{5}$ & SU & SU & & & \\
\hline \multirow[t]{4}{*}{4} & & 1.63 & 2.40 & 2.80 & 3.61 & 4.00 & 4.23 & 4.59 & 4.98 \\
\hline & & 1.62 & 2.38 & 2.97 & (3.63) & 4.04 & 4.10 & 4.70 & \\
\hline & $1^{2} B_{2 u}$ & $1^{1} A_{g}$ & $1^{3} B_{1 g}$ & ${ }^{1}{ }^{1} B_{1 g}$ & $\left(1^{3} B_{3 u}\right)$ & $1^{3} B_{2 g}$ & $1{ }^{3} B_{2 u}$ & $1^{1} B_{2 u}$ & \\
\hline & $1 s^{2} 1 p^{3}$ & $1 p_{2}^{-1}$ & $1 p_{1}^{-1}(\mathrm{~T})$ & $1 p_{1}^{-1}(\mathrm{~S})$ & Frag? & $\mathrm{SU}$ & $1 s^{-1}(\mathrm{~T})$ & $1 s^{-1}(\mathrm{~S})$ & \\
\hline \multirow[t]{4}{*}{5} & & 2.12 & 3.32 & 3.76 & 4.09 & 4.36 & 4.58 & & \\
\hline & & 2.04 & 3.32 & 3.92 & 4.28 & 4.44 & & & \\
\hline & ${ }^{1} A_{1}$ & $1^{2} A_{1}$ & $1^{2} B_{2}$ & $2{ }^{2} A_{1}$ & $2^{2} B_{2}$ & $1^{2} B_{1}$ & & & \\
\hline & $1 s^{2} 1 p^{4}$ & $1 p_{2}^{-1}$ & $1 p_{1}^{-1}$ & SU & $\mathrm{SU}$ & $\mathrm{SU}$ & $1 s^{-1}$ & & \\
\hline \multirow[t]{4}{*}{6} & & 2.08 & 2.36 & 2.66 & 3.19 & 3.53 & 3.85 & 4.25 & \\
\hline & & 2.12 & 2.39 & 2.86 & 3.29 & 3.83 & & & \\
\hline & $1{ }^{2} B_{1}$ & $1^{1} A_{1}$ & $1^{3} B_{1}$ & $1{ }^{1} B_{1}$ & $1{ }^{3} A_{2}$ & $1^{1} A_{2}$ & & & \\
\hline & $1 s^{2} 1 p^{5}$ & $1 p_{3}^{-1}$ & $1 p_{2}^{-1}(\mathrm{~T})$ & $1 p_{2}^{-1}(\mathrm{~S})$ & $1 p_{1}^{-1}(\mathrm{~T})$ & $1 p_{1}^{-1}(\mathrm{~S})$ & SU & SU & \\
\hline \multirow[t]{2}{*}{7} & & 2.60 & 2.73 & 3.60 & & & & & \\
\hline & $1 s^{2} 1 p^{6}$ & $1 p_{3}^{-1}$ & $1 p_{2}^{-1}$ & $1 p_{1}^{-1}$ & & & & & \\
\hline 8 & & 1.61 & 3.06 & 3.18 & 3.97 & & & & \\
\hline & & 1.46 & 3.18 & (3.38) & 4.21 & & & & \\
\hline & $1^{2} A_{1}$ & ${ }^{1} A_{1}$ & $1{ }^{3} E$ & $1{ }^{3} E$ & $2{ }^{3} B_{2}$ & (Only trip & itions lis & & \\
\hline & $1 s^{2} 1 p^{6} 1 d^{1}$ & $1 d_{1}^{-1}$ & $1 p_{3}^{-1}$ & $1 p_{2}^{-1}$ & $1 p_{1}^{-1}$ & (Singlet/t & itting $<\mathrm{F}$ & & \\
\hline 9 & & 2.45 & 2.77 & 3.19 & 3.33 & 4.44 & 4.75 & 5.81 & \\
\hline & $1 s^{2} 1 p^{6} 1 d^{2}$ & Isomer & $1 d_{1}^{-1}$ & $1 p_{3}^{-1}$ & $1 p_{2}^{-1}$ & $1 p_{1}^{-1}$ & $\mathrm{SU}$ & $1 s^{-1}$ & \\
\hline 10 & & 2.13 & 2.80 & 3.09 & 3.50 & 3.81 & 4.90 & & \\
\hline & $1 s^{2} 1 p^{6} 1 d^{3}$ & $1 d_{2}^{-1}$ & $1 d_{1}^{-1}$ & $1 p_{3}^{-1}$ & $\mathrm{SU}$ & $1 p_{2}^{-1}$ & $1 p_{1}^{-1}$ & & \\
\hline 11 & & 2.18 & 2.53 & 3.07 & 3.26 & 4.10 & 4.27 & 4.46 & 5.35 \\
\hline & $1 s^{2} 1 p^{6} 1 d^{4}$ & Isomer & $1 d_{2}^{-1}$ & $1 d_{1}^{-1}$ & $1 p_{3}^{-1}$ & $1 p_{2}^{-1}$ & $\mathrm{SU}$ & $\mathrm{SU}$ & $1 p_{1}^{-1}$ \\
\hline 12 & & 2.18 & 2.91 & 3.12 & 3.42 & 3.62 & 4.58 & & \\
\hline & $1 s^{2} 1 p^{6} 1 d^{5}$ & $1 d_{3}^{-1}$ & $1 d_{2}^{-1}$ & $1 d_{1}^{-1}$ & $1 p_{3}^{-1}$ & $\mathrm{SU}$ & $1 p_{2}^{-1}$ & & \\
\hline 13 & & 2.10 & 2.91 & 3.13 & 3.40 & 3.60 & 5.05 & & \\
\hline & $1 s^{2} 1 p^{6} 1 d^{6}$ & Isomer & $1 d_{3}^{-1}$ & $1 d_{2}^{-1}$ & $1 d_{1}^{-1}$ & $1 p_{3}^{-1}$ & $1 p_{2}^{-1}$ & & \\
\hline 14 & & 2.12 & 3.09 & 3.18 & 3.35 & 3.54 & 3.88 & 5.30 & \\
\hline & $1 s^{2} 1 p^{6} 1 d^{7}$ & $1 d_{4}^{-1}$ & $1 d_{3}^{-1}$ & SU & $1 d_{2}^{-1}$ & $1 d_{1}^{-1}$ & $1 p_{3}^{-1}$ & $1 p_{2}^{-1}$ & \\
\hline 15 & & 2.58 & 2.69 & 3.19 & 3.40 & 3.62 & 4.25 & & \\
\hline & $1 s^{2} 1 p^{6} 1 d^{8}$ & $1 d_{4}^{-1}$ & Isomer & $1 d_{3}^{-1}$ & $1 d_{2}^{-1}$ & $1 d_{1}^{-1}$ & $1 p_{3}^{-1}$ & & \\
\hline 16 & & 2.45 & 2.95 & 3.30 & 3.45 & 3.78 & & & \\
\hline & $1 s^{2} 1 p^{6} 1 d^{9}$ & $1 d_{5}^{-1}$ & $1 d_{4}^{-1}$ & $1 d_{3}^{-1}$ & $1 d_{2}^{-1}$ & $1 d_{1}^{-1}$ & & & \\
\hline 17 & & 3.12 & 3.20 & 3.29 & 3.70 & 3.80 & & & \\
\hline & $1 s^{2} 1 p^{6} 1 d^{10}$ & $\left(1 d_{5}^{-1} 1 d\right.$ & ${ }^{1} 1 d_{3}^{-1} 1 d_{2}^{-}$ & & $\mathrm{SU}$ & $\mathrm{SU}$ & & & \\
\hline 18 & & 2.58 & 3.31 & 3.50 & 3.73 & 3.87 & 4.07 & & \\
\hline & $(s p d) 2 s^{1}$ & $2 s^{-1}$ & $1 d_{5}^{-1}$ & $1 d_{4}^{-1}$ & $1 d_{3}^{-1}$ & $1 d_{2}^{-1}$ & $1 d_{1}^{-1}$ & & \\
\hline 19 & & 2.84 & 3.39 & 3.56 & 3.74 & 4.10 & & & \\
\hline & $(s p d) 2 s^{2}$ & $2 s^{-1}$ & $1 d_{5}^{-1}$ & $1 d_{4}^{-1}$ & $1 d_{3}^{-1}$ & $1 d_{2}^{-1} 1 d_{1}^{-}$ & & & \\
\hline 20 & & 2.31 & 3.00 & 3.40 & 3.79 & $4.10-4.6$ & & & \\
\hline & $(s p d) 2 s^{2} 1 f^{1}$ & $1 f_{1}^{-1}$ & $2 s^{-1}$ & $1 d_{5}^{-1}$ & $1 d_{4}^{-1}$ & $1 d_{3}^{-1} 1 d_{2}^{-}$ & & & \\
\hline 21 & & 2.84 & 3.04 & 3.46 & 3.84 & 4.08 & 4.33 & & \\
\hline & $(s p d) 2 s^{2} 1 f^{2}$ & $1 f_{1}^{-1}$ & $2 s^{-1}$ & $1 d_{5}^{-1}$ & $1 d_{4}^{-1}$ & $1 d_{3}^{-1}$ & $1 d_{2}^{-1}$ & & \\
\hline
\end{tabular}




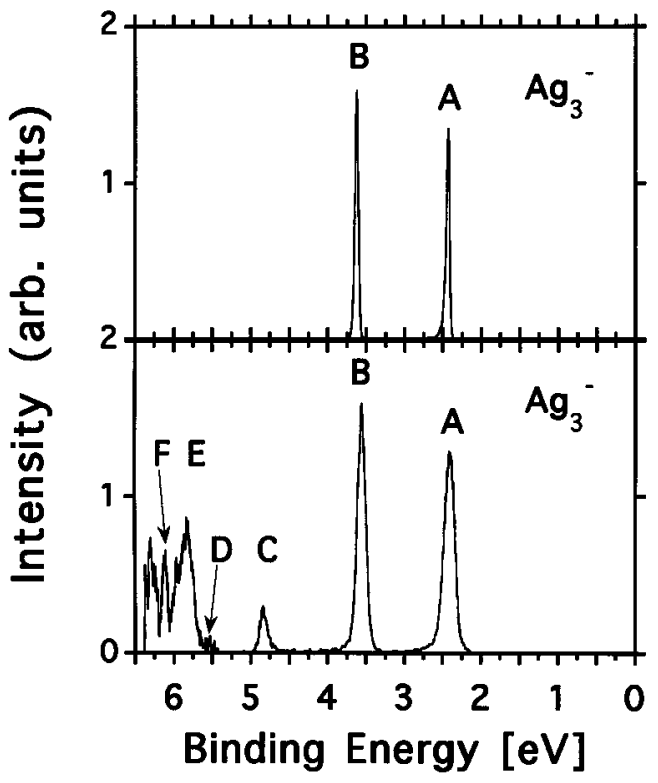

FIG. 4. Photoelectron spectra of $\mathrm{Ag}_{3}^{-}$taken with $h \nu=6.424 \mathrm{eV}$ and $h \nu$ $=4.025 \mathrm{eV}$, respectively. For the assignment of the marked features (A, B, $\mathrm{C}, \mathrm{D}, \mathrm{E}$, and F) see the text.

unpaired electrons in the neutral dimer. The two electrons can combine to a singlet and a triplet state resulting in two peaks ( $\mathrm{B}$ and $\mathrm{C}$ ) corresponding to the photoemission out of the $\sigma$ orbital. In the case of the dimer the singlet/triplet splitting is extremely large $(1.1 \mathrm{eV})$. The multiplet splitting decreases with increasing cluster size, because the average distance of the interacting electrons increases.

Feature A can be assigned to photoemission of the single electron occupying the antibonding $\sigma^{*}$ orbital in the anion, while features $\mathrm{B}$ and $\mathrm{C}$ are assigned to the multiplet corresponding to the photoemission from the bonding $\sigma$ orbital. The electronic shell model predicts a configuration $1 s^{2} 1 p^{1}$ for $\mathrm{Ag}_{2}^{-}$. From the point of view of the molecular symmetry the $1 s$ shell has a certain similarity with the bonding $\sigma$ orbital, while the $1 p$ shell exhibits the same number of nodes as the $\sigma^{*}$ orbital.

$\mathrm{Ag}_{3}^{-}$

Photoelectron spectra of $\mathrm{Ag}_{3}^{-}$taken with $h \nu=6.424 \mathrm{eV}$ and $h \nu=4.025 \mathrm{eV}$ are displayed in Fig. 4. The two spectra clearly demonstrate the variation of the energy resolution with the kinetic energy of the electrons. Compared to the features observed in the spectrum of $\mathrm{Ag}_{2}^{-}$the linewidth of the ground state transition (marked $\mathrm{A})$ is relatively narrow $(<50$ $\mathrm{meV}$ compared to $100 \mathrm{meV}$ for peaks $\mathrm{A}$ and $\mathrm{B}$ of $\mathrm{Ag}_{2}$ ) indicating a relatively small geometry change induced by the detachment.

According to the calculations $\mathrm{Ag}_{3}^{-}$is linear, while neutral $\mathrm{Ag}_{3}$ is strongly bent. ${ }^{10}$ In general, such a large difference in geometry gives rise to a major difficulty concerning the interpretation of photoelectron spectra of negatively charged particles: The spectra contain information about the neutral vibronic states of the cluster in the geometry of the electronic ground state of the anion, because the photoemission process is fast compared to the movements of the nuclei. Therefore, the final states (=electronic states of neutral $\mathrm{Ag}_{3}$ ) given in
Table I $\left({ }^{2} \Sigma_{u}^{+}\right)$refer to the linear isomer, which is not the most stable (for details see Refs. 10 and 24). With increasing cluster size the difference in geometry between a neutral and a charged metal cluster may decrease, because the charge is distributed over an increasing number of atoms.

The electronic ground state of $\mathrm{Ag}_{3}^{-}$with four valence electrons is ${ }^{1} \Sigma_{g}^{+}$. Within a BE range of $6 \mathrm{eV}$ the existence of five electronic transitions into the neutral final states ${ }^{2} \Sigma_{u}^{+},{ }^{2} \Sigma_{g}^{+}, 2{ }^{2} \Sigma_{g}^{+},{ }^{2} \Pi_{g}^{+}, 2{ }^{2} \Sigma_{u}^{+}$is predicted. The calculated BE's match within $0.1 \mathrm{eV}$ the positions of the peaks A-E (see Table I). All calculated excited states involve the excitation of $4 s$ electrons only with no contributions from $4 d$ electrons in agreement with the above considerations.

The three $s$ orbitals of the trimer combine to three molecular orbitals: the bonding $\sigma_{g}$, the nonbonding $\sigma_{u}$, and the antibonding $2 \sigma_{g}$. The anion ground state ${ }^{1} \Sigma_{g}^{+}$can be described by the configuration $\sigma_{g}^{2} \sigma_{u}^{2}$. The linear neutral ground state ${ }^{2} \Sigma_{u}^{+}$has the configuration $\sigma_{g}^{2} \sigma_{u}$. Therefore, feature A can be assigned to photoemission from the nonbonding $\sigma_{u}$ orbital. The nonbonding character of this orbital can also explain the relatively narrow linewidth of feature A. The first excited state ${ }^{2} \Sigma_{g}^{+}$has the leading configuration $\sigma_{g} \sigma_{u}^{2}$. Peak B can be assigned to photoemission from the bonding $\sigma_{g}$ orbital. Within the simple model of photoemission the two dominant features A and B mirror the two occupied single particle orbitals of the trimer.

However, the first excited state (peak B) has a contribution $(13 \%)$ from the configuration $\sigma_{g}^{2} 2 \sigma_{g}$. This configuration corresponds to an excitation of the single remaining electron in the $\sigma_{u}$ orbital into the unoccupied antibonding $2 \sigma_{g}$ orbital accompanying the detachment of a $\sigma_{u}$ electron. Therefore, the single particle picture fails in the accurate description of even the first excited state of the trimer. However, the leading configuration has a contribution of more than $70 \%$ to this state and fits well into the simple picture. For a qualitative understanding of the data this limited accuracy might be sufficient.

Within the simple picture no other features at higher BE are expected. The qualitative understanding of these peaks $(\mathrm{C}-\mathrm{F})$ is only possible, if further shake-up processes are taken into account. Feature $\mathrm{C}$ is assigned to the $2{ }^{2} \Sigma_{g}^{+}$state with a leading configuration $\sigma_{g}^{2} 2 \sigma_{g}$. This is the configuration according to the excitation $\sigma_{u} \rightarrow 2 \sigma_{g}$ which also contributes to the ${ }^{2} \Sigma_{g}^{+}$state discussed above. Correspondingly, this state has also a contribution from the $\sigma_{g} \sigma_{u}^{2}$ configuration. Therefore, feature $\mathrm{C}$ can be assigned to a shake-up peak.

According to the calculations, the weak feature D is assigned to a transition into a ${ }^{2} \Pi_{g}^{+}$state. The leading configuration involves the occupation of the $\pi_{u}$ orbital. Therefore, this peak corresponds to a transition involving the excitation into an even higher unoccupied orbital, which might explain the relatively low intensity.

Feature $\mathrm{E}$ is assigned to the transition into the $2^{2} \Sigma_{u}^{+}$ state with a leading configuration $\sigma_{g} \sigma_{u} 2 \sigma_{g}(40 \%)$. This transition can be interpreted as a $\sigma_{g} \rightarrow 2 \sigma_{g}$ shake up with respect to the direct photoemission peak A. However, other configurations contribute with considerable relative weights to this excited state.

From the above analysis a qualitative picture of the spec- 


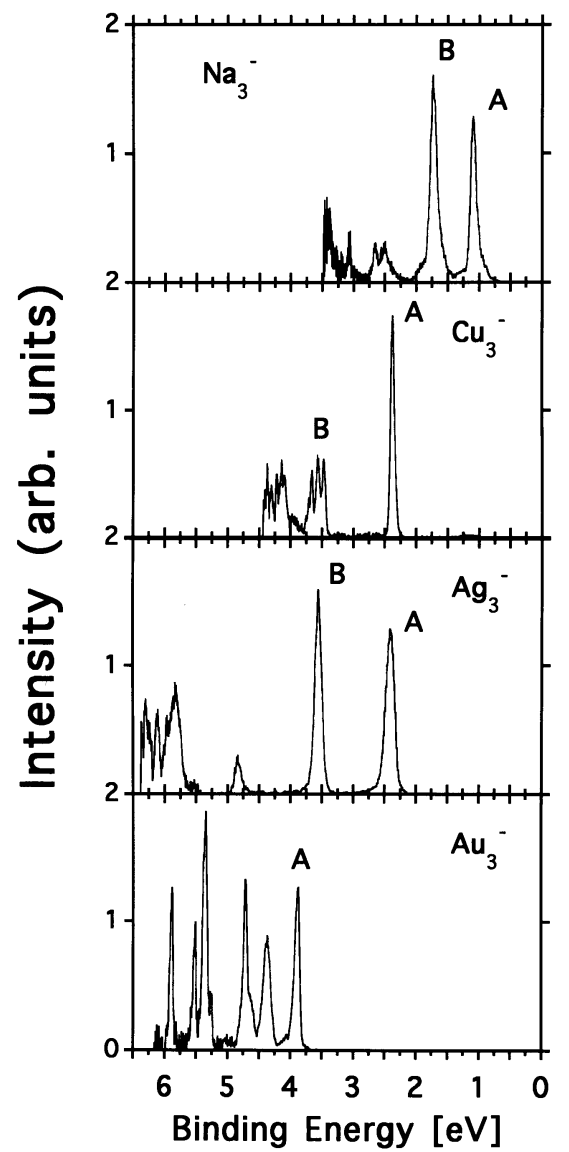

FIG. 5. Comparison of photoelectron spectra of $\mathrm{Na}_{3}^{-}, \mathrm{Cu}_{3}^{-}, \mathrm{Ag}_{3}^{-}$, and $\mathrm{Au}_{3}^{-}$. The spectra of $\mathrm{Ag}_{3}^{-}$and $\mathrm{Au}_{3}^{-}$are recorded with $h \nu=6.424 \mathrm{eV}$ photon energy, the spectrum of $\mathrm{Na}_{3}^{-}$with $h \nu=3.49 \mathrm{eV}$ and the spectrum of $\mathrm{Cu}_{3}^{-}$with $h \nu=5.0 \mathrm{eV}$ photon energy. For a discussion of the marked features (A and B) see the text.

trum of $\mathrm{Ag}_{3}^{-}$emerges, if only the leading configurations are taken into consideration: Features $\mathrm{A}$ and $\mathrm{B}$ can be assigned to direct photoemission from the occupied bonding and nonbonding orbitals. Both peaks are accompanied by a shake-up feature of an electron into the antibonding orbital. Additional features can be assigned to shake-up processes into higher orbitals.

In terms of the shell model the configuration of $\mathrm{Ag}_{3}^{-}$is $1 s^{2} 1 p^{2}$. The symmetries of the $1 s$ and $1 p$ shells correspond to the symmetries of the $\sigma_{g}$ and $\sigma_{u}$ orbitals. Qualitatively, the configuration $1 s^{2} 1 p^{2}$ corresponds to the configuration $\sigma_{g}^{2} \sigma_{u}^{2}$. The two dominant peaks in the spectrum of $\mathrm{Ag}_{3}^{-}$can be assigned to the photoemission from the $1 s$ and $1 p$ shells, respectively.

Figure 5 displays a comparison of the photoelectron spectra of $\mathrm{Na}_{3}^{-}, \mathrm{Cu}_{3}^{-}, \mathrm{Ag}_{3}^{-}$, and $\mathrm{Au}_{3}^{-}$. The spectra of $\mathrm{Ag}_{3}^{-}$ and $\mathrm{Au}_{3}^{-}$are both recorded with $h \nu=6.424 \mathrm{eV}$ photon energy, the spectrum of $\mathrm{Na}_{3}^{-}$with $h \nu=3.49 \mathrm{eV}$ and the spectrum of $\mathrm{Cu}_{3}^{-}$with $h \nu=5.0 \mathrm{eV}$ photon energy.

According to calculations ${ }^{23,24}$ and experimental results $2,6,10,18,26$ the four trimer anions are linear. Therefore, any difference in the photoelectron spectra must be due to differences in the electronic structure of the four monovalent metals. Such differences have been already discussed for the bulk density of states (see Fig. 1). A large similarity of the electronic structure of $\mathrm{Ag}$ and $\mathrm{Na}$ is expected, if a scaling factor for the difference in the overall bandwidth is taken into account. Indeed, the spectra of $\mathrm{Na}_{3}^{-}$and $\mathrm{Ag}_{3}^{-}$are similar, if the $\mathrm{BE}$ scale of the spectrum of $\mathrm{Na}_{3}^{-}$is expanded by a factor of 2. The two dominant peaks (marked A and B) are assigned to corresponding transitions $\left({ }^{2} \Sigma_{u}^{+} \leftarrow{ }^{1} \Sigma_{g}^{+}\right.$; $\left.{ }^{2} \Sigma_{g}^{+} \leftarrow{ }^{1} \Sigma_{g}^{+}\right)$. The $s$-derived states of $\mathrm{Cu}$ and $\mathrm{Ag}$ are expected to be similar. This is supported by the observation of two features $(\mathrm{A}$ and $\mathrm{B})$ at about the same $\mathrm{BE}$ for both trimers. The splitting of peak $\mathrm{B}$ in the spectrum of $\mathrm{Cu}_{3}^{-}$can be explained by a partial hybridization with $3 d$-derived orbitals, which are located at slightly higher $\mathrm{BE}(0.3 \mathrm{eV}){ }^{18}$

The VDE of $\mathrm{Au}_{3}^{-}(3.89 \mathrm{eV})$ is much larger than the VDE's of $\mathrm{Cu}_{3}^{-}(2.37 \mathrm{eV})$ and $\mathrm{Ag}_{3}^{-}(2.43 \mathrm{eV})$, a general trend observed for all Au clusters and the atom. In Au and other heavy metals the $6 s$ orbitals are contracted and stabilized by relativistic effects with respect to $\mathrm{Cu}$ and $\mathrm{Ag}$. This effect enhances the binding energy of an additional electron to a $\mathrm{Au}$ cluster, while the ionization potential of the $\mathrm{Au}$ atom and the bulk work function (Fig. 1) are only slightly larger than the values for $\mathrm{Ag}$ and $\mathrm{Cu}$. We know of no simple explanation, why the relativistic contraction has a larger effect on the electron affinity than on the ionization potential.

For $\mathrm{Au}_{3}^{-}$this shift of the $6 s$-derived orbitals locates the second $s$-derived feature (corresponding to the transition ${ }^{2} \Sigma_{g}^{+} \leftarrow{ }^{1} \Sigma_{g}^{+}$) within the manifold of transitions into $5 d_{5 / 2}$ derived states. A strong hybridization of the ${ }^{2} \Sigma_{g}^{+}$state with the $5 d_{5 / 2}$ derived states is expected to occur and an unambiguous assignment of these transitions (unmarked features in the spectrum of $\mathrm{Au}_{3}^{-}$) is not possible at present. ${ }^{26}$

The spectra of the trimers correspond to the scheme of the electronic structure of the bulk metals in Fig. 1 and especially demonstrate the correspondence of the alkali metals and $\mathrm{Ag}$. However, for larger clusters this similarity is less well pronounced, since these clusters exhibit differences in their geometric structure due to the influence of the $4 d$ orbitals.

$\mathrm{Ag}_{4}^{-}$

Figure 6 shows photoelectron spectra of $\mathrm{Ag}_{4}^{-}$recorded at four different photon energies: $h \nu=4.025,4.66,5.0$, and $6.424 \mathrm{eV}$. Eleven transitions $(\mathrm{A}-\mathrm{K})$ are identified in the spectra (A-H listed in Table I). The comparison demonstrates the additional information about a cluster gained by recording photoelectron spectra at higher photon energies. The spectrum at $h \nu=4.0 \mathrm{eV}$ is almost identical with the pioneering high resolution studies from Ho et al. ${ }^{10}$ The FWHMs of features $\mathrm{B}(70 \mathrm{meV})$ and $\mathrm{C}(60 \mathrm{meV})$ are slightly smaller than in the earlier work (100 and $110 \mathrm{meV}$, respectively), which might be due to the lower vibrational temperature of the anions.

The changes in the relative intensities of the peaks visible in the different spectra correspond to the changes in cross section at different photon energies. Feature D (BE $=3.61 \mathrm{eV}$ ) displays a thresholdlike behavior. In the spectra recorded with $h \nu=4.025 \mathrm{eV}$ and $h \nu=4.66 \mathrm{eV}$ it is below the limit of detection, whereas the relative intensity is approximately constant at $h \nu=5.0 \mathrm{eV}$ and $h \nu=6.424 \mathrm{eV}$. Such behavior is not expected for direct photodetachment peaks, 


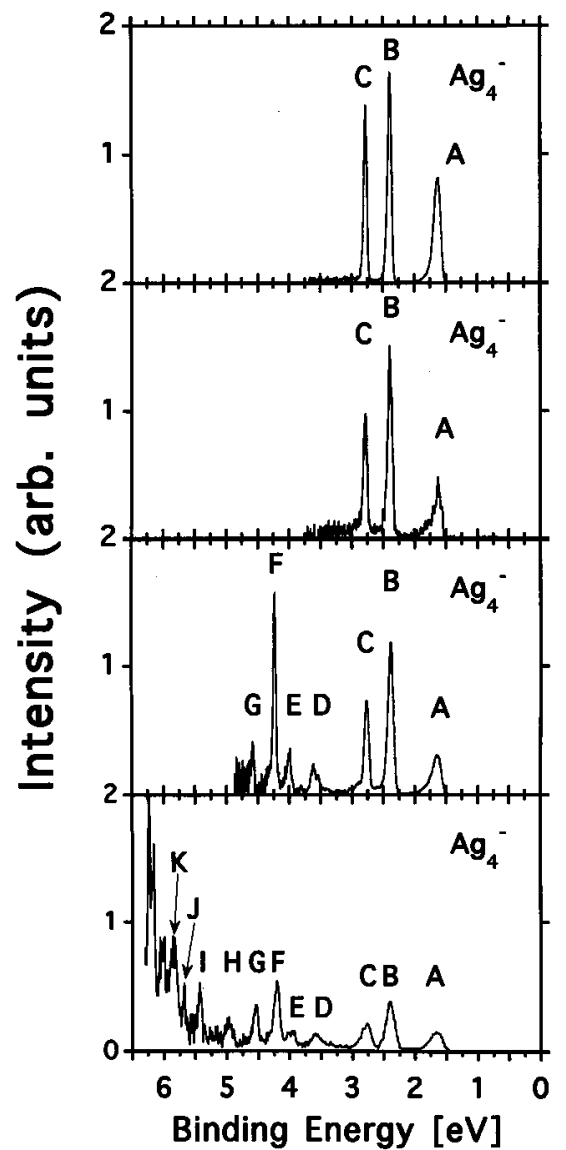

FIG. 6. Photoelectron spectra of $\mathrm{Ag}_{4}^{-}$recorded at $h \nu=4.025,4.66,5.0$, and $6.424 \mathrm{eV}$, respectively. The marked features $(\mathrm{A})-(\mathrm{K})$ are discussed in the text.

since the detachment cross section usually increases monotonically close to the threshold $(=3.61 \mathrm{eV})$.

A possible explanation for the appearance of peak $\mathrm{D}$ is fragmentation into $\mathrm{Ag}_{3}^{-}$. The binding energy of peak $\mathrm{D}$ is essentially equal to the one of feature $\mathrm{B}$ in the spectrum of $\mathrm{Ag}_{3}^{-}$. If there is a fast (with respect to the pulse length of the laser $=10-20 \mathrm{~ns}$ ) fragmentation process $\mathrm{Ag}_{4}^{-} \rightarrow \mathrm{Ag}_{3}^{-}$, which opens up for $h \nu>4.66 \mathrm{eV}$, a photoelectron spectrum of $\mathrm{Ag}_{3}^{-}$ is superimposed onto the spectrum of $\mathrm{Ag}_{4}^{-}$. Feature $\mathrm{A}$ of the spectrum of $\mathrm{Ag}_{3}^{-}$overlaps with the strong peak $\mathrm{B}$ of $\mathrm{Ag}_{4}^{-}$and is therefore not visible. The relative intensity of the fragment photoelectron spectrum depends on the photodetachment cross sections of $\mathrm{Ag}_{3}^{-}$and $\mathrm{Ag}_{4}^{-}$and the photofragmentation cross section of $\mathrm{Ag}_{4}^{-}$. The $\mathrm{Ag}_{3}^{-}$fragment is probably vibrationally excited, which might explain the larger linewidth of peak D compared to peak $\mathrm{B}$ in the spectrum of $\mathrm{Ag}_{3}^{-}$. The relative intensity of feature $\mathrm{D}$ does not change with laser intensity, as it might be expected if it is due to a two-photon process. However, if the cross sections for the two steps involved in two-photon process are largely different, the smaller one determines the yield resulting in a linear intensity dependence. Therefore, a linear dependence is not a proof for a one-photon process. According to these considerations feature $\mathrm{D}$ is tentatively assigned to $\mathrm{Ag}_{3}^{-}$. No other fragmentation features are observed in the data on any of the other $\mathrm{Ag}_{n}^{-}$clusters investigated here.
According to the calculations, $\mathrm{Ag}_{4}^{-}$is a rhombus with $D_{2 h}$ symmetry. The calculated peak positions of the photoelectron spectra of $\mathrm{Ag}_{4}^{-}$are listed in Table I. Feature A is assigned to the transition from the ground state of the anion ${ }^{2} B_{2 u}$ into the neutral ground state ${ }^{1} A_{g}$. There is a relatively large number of features, which can be assigned to transitions into various predicted electronic states of the neutral. For the features at BE's $>4 \mathrm{eV}$ an unambiguous assignment due to the position only is not possible. However, based on the assumption that a shake-up feature should exhibit a smaller intensity than a direct emission peak a tentative assignment is presented here.

The five $s$ electrons of $\mathrm{Ag}_{4}^{-}$occupy the three lowest $s$-derived orbitals with the configuration $a_{g}^{2} b_{3 u}^{2} b_{2 u}$. The configuration of the neutral ground state is $a_{g}^{2} b_{3 u}^{2}$. Therefore, feature A can be assigned to the direct emission of the uppermost unpaired electron of the anion. Feature $B$ has to be assigned to the transition into the ${ }^{3} B_{1 g}$ state, since the calculated $\mathrm{BE}$ is almost identical with the measured one and no other calculated state is close in BE. The configuration is $a_{g}^{2} b_{3 u}^{1} b_{2 u}^{1}$. Therefore, this feature is assigned to the triplet state resulting from the direct photoemission of one of the two electrons of the $b_{3 u}$ orbital. For intensity reasons (singlet:triplet $=1: 3$ ) and because of the agreement in $\mathrm{BE}$ peak $C$ is assigned to the transition into the ${ }^{1} B_{1 g}$ state. The experimentally determined singlet/triplet intensity ratio is in most cases (see also Ref. 18) too large (about 1:2) and depends on the photon energy. The reason for this is not known. The ${ }^{1} B_{1 g}$ state is the singlet state resulting from the photoemission from the $b_{3 u}$ orbital. As in the case of the dimer, the singlet-triplet splitting is slightly overestimated in the calculation (exp. $0.4 \mathrm{eV}$, theory $0.57 \mathrm{eV}$ ).

Analogously, at higher BE the appearance of a singlet/ triplet doublet is expected corresponding to the photoemission from the $a_{g}$ orbital. The configuration of these excited states would be $a_{g}^{1} b_{3 u}^{2} b_{2 u}^{1}$. A possible doublet of peaks with a separation of about $0.4 \mathrm{eV}$ and an intensity ratio similar to the ratio of $\mathrm{B} / \mathrm{C}$ are the features $\mathrm{F}$ and $\mathrm{G}$ (BE's=4.23 and $4.59 \mathrm{eV}$ ). However, there are no calculated states with a pure $a_{g}^{1} b_{3 u}^{2} b_{2 u}^{1}$ configuration. The hole in the $a_{g}$ orbital corresponds to a significant excitation of the cluster, thus disturbing all valence orbitals, which results in a strong configuration mixing. The calculation gives a ${ }^{3} B_{2 u}$ state $(\mathrm{BE}=4.10$ $\mathrm{eV}$ ) with the correct leading configuration $(40 \%)$, but also considerable contributions from several other configurations. Since there is no other triplet state in this BE range except for pure shake-up states, we assign the intense feature $\mathrm{F}$ to the ${ }^{3} B_{2 u}$ state. The corresponding ${ }^{1} B_{2 u}$ state is predicted to be at $4.70 \mathrm{eV}$ BE. Thus if the calculated BE of the ${ }^{1} B_{2 u}$ is corrected for the $-0.15 \mathrm{eV}$ deviation of the triplet state and the systematic overestimation of the calculated singlet/triplet splitting the assignment of feature $\mathrm{G}$ to the ${ }^{1} B_{2 u}$ state seems reasonable.

Beside these five features, which at least partially correspond to direct photoemission from the three occupied valence orbitals, several shake-up processes result in the appearance of various additional peaks. In between the two doublets $(\mathrm{B} / \mathrm{C}$ and $\mathrm{F} / \mathrm{G})$ two small features are visible (D: $\mathrm{BE}=3.61 \mathrm{eV}, \mathrm{E}: \mathrm{BE}=4.00 \mathrm{eV})$. Because of its unusual, 


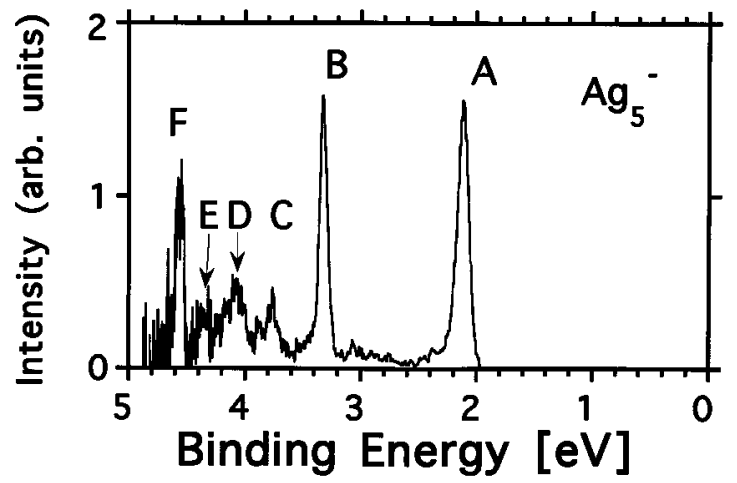

FIG. 7. Photoelectron spectrum of $\mathrm{Ag}_{5}^{-}$recorded at $h \nu=5.0 \mathrm{eV}$. For a discussion of the marked features $(\mathrm{A})-(\mathrm{F})$ see the text.

threshold like photon energy dependence feature $\mathrm{D}$ has above been assigned to the emission from $\mathrm{Ag}_{3}^{-}$fragments. At the same energy $(\mathrm{BE}=3.63 \mathrm{eV})$ the calculation predicts a transition into a ${ }^{3} B_{3 u}$ state. The configuration of this state is $a_{g}^{2} b_{3 u}^{1} 2 a_{g}^{1}$. Such a transition involves an electron excitation. Although the BE's of the predicted transition and the peak position fit almost perfectly, it is difficult to consider a photodetachment process with the observed photon energy dependence. Feature E can be assigned to the transition into the ${ }^{3} B_{2 g}$ state $(\mathrm{BE}=4.04 \mathrm{eV})$ with the configuration $a_{g}^{2} b_{3 u}^{1} b_{1 u}^{1}$. This is also a shake-up feature. The low intensity of this feature supports the primary assumption of lower intensities of shake-up peaks.

There are other features at higher BE (H, I, J, and K), which can also be tentatively assigned to various shake-up transitions. However, at about $6 \mathrm{eV} \mathrm{BE}$ photoemission from $4 d$ orbitals is expected to contribute significantly to the photoelectron spectra and therefore the assignments of features $\mathbf{J}$ and $\mathrm{K}$ to $4 s$-derived states seems tentative. The relatively high intensity of peak $\mathrm{K}$ may serve as an indication for the onset of $4 d$ emission.

In terms of the shell model the configuration of $\mathrm{Ag}_{4}^{-}$is $1 s^{2} 1 p^{3}$. If the particle is not spherical, the $p$ orbital will split into sublevels of different energies: $1 s^{2} 1 p_{1}^{2} 1 p_{2}^{1}$. According to the simple model of photoemission including multiplet splitting five peaks are expected in the photoelectron spectra: a single peak for the emission of the $1 p_{2}^{1}$ electron and a doublet for the two fully occupied subshells. According to the above considerations the doublets, F/G and B/C can be assigned to the emission from the $1 s$ and $1 p_{1}$ shells, respectively, and the single peak A corresponds to the $1 p_{2}$ shell. Within the $D_{2 h}$ point group the symmetry of the $a_{g}, b_{3 u}$, and $b_{2 u}$ orbitals corresponds exactly to the symmetry of the $1 \mathrm{~s}$, $1 p_{1}$, and $1 p_{2}$ electronic subshells.

$\mathrm{Ag}_{5}^{-}$

Figure 7 displays a photoelectron spectrum of $\mathrm{Ag}_{5}^{-}$recorded at $h \nu=5.0 \mathrm{eV}$ photon energy. No photoelectron spectra could be recorded at $h \nu=6.424 \mathrm{eV}$ photon energy for $\mathrm{Ag}_{5}^{-}$and $\mathrm{Ag}_{6}^{-}$due to their low abundance in the mass spectra. Six transitions (marked A-F, see Table I) can be identified. The linewidths of features $\mathrm{A}$ and $\mathrm{B}$ are large compared to the experimental resolution. Therefore, the photoelectron spectrum recorded at $h \nu=4.025 \mathrm{eV}$ contains no additional information and is not shown.

According to the calculations, the pentamer anion is planar $\left(C_{2 v}\right.$ symmetry). The electronic ground state of the anion is ${ }^{1} A_{1}$. Five observed peaks $(\mathrm{A}-\mathrm{E})$ can be assigned to transitions into the electronic ground state ${ }^{2} A_{1}(\mathrm{~A})$ and four excited states ${ }^{2} B_{2}, 2{ }^{2} A_{1}, 2{ }^{2} B_{2},{ }^{2} B_{1}$ (B, C, D, and E) of the neutral, respectively. The calculated BE's of the transitions are listed in Table I. The agreement between the calculation and the experiment is less well pronounced than for the smaller clusters but in all cases better than $0.2 \mathrm{eV}$.

The pentamer anion has six $s$ electrons occupying three single particle orbitals in the configuration $a_{1}^{2} b_{2}^{2} 2 a_{1}^{2}$. The configuration of the neutral ground state ${ }^{2} A_{1}$ is $a_{1}^{2} b_{2}^{2} 2 a_{1}^{1}$. Therefore, feature A can be assigned to direct photoemission from the $2 a_{1}$ orbital. The first excited state ${ }^{2} B_{2}$ has a leading (79\%) configuration $a_{1}^{2} b_{2}^{1} 2 a_{1}^{2}$. Therefore, feature B corresponds to photoemission from the $b_{2}$ orbital. The relatively low intensities of peaks C, D, and E indicate shake-up processes responsible for the appearance of these features. Indeed, the leading configurations of the calculated neutral states $2{ }^{2} A_{1}, 2{ }^{2} B_{2}$, and ${ }^{2} B_{1}$ located at about the same BE's (see Table I) differ from the configuration of the anion by more than one changed occupation number corresponding to shake-up processes. Since calculations for neutral excited states at higher binding energies are not available, feature $\mathrm{F}$ cannot be assigned. However, due to its relatively high intensity indicating a direct emission process we tentatively assign this feature to a transition into a doublet state with the leading configuration $a_{1}^{1} b_{2}^{2} 2 a_{1}^{2}$. Accordingly, this peak corresponds to emission from the $a_{1}$ orbital. Analogous to the tetramer this highly excited state is probably strongly perturbed and might not be described by a single configuration only.

According to the above considerations the three intense features in the photoelectron spectrum of $\mathrm{Ag}_{5}^{-}$mirror the three occupied single particle orbitals. Since the anion ground state is a singlet state, a multiplet splitting is not expected and the number of intense features corresponds to the number of orbitals.

In terms of the ellipsoidal shell model the configuration of $\mathrm{Ag}_{5}^{-}$is $1 s^{2} 1 p_{1}^{2} 1 p_{2}^{2}$. Therefore, the three intense photoemission peaks ( $\mathrm{A}, \mathrm{B}$, and $\mathrm{F}$ ) can be tentatively assigned to the photoemission from these three subshells. If this assignment is correct, the quantum chemical single particle orbitals $a_{1}, b_{2}$, and $2 a_{1}$ correspond to the subshells $1 s, 1 p_{1}$, and $1 p_{2}$, respectively. Indeed, these orbitals have the equivalent symmetries in the $C_{2 v}$ point group supporting qualitatively the shell model. Also, the shell model predicts an oblate deformation of this cluster in qualitative agreement with the calculated planar structure of the pentamer anion.

$\mathrm{Ag}_{6}^{-}$

Figure 8 shows a comparison of photoelectron spectra of $\mathrm{Ag}_{6}^{-}$recorded at $h \nu=5.0 \mathrm{eV}$ and $h \nu=4.025 \mathrm{eV}$ photon energy with a photoelectron spectrum of $\mathrm{Cu}_{6}^{-}$taken at $h \nu=5.0$ $\mathrm{eV}$. Seven features $(\mathrm{A}-\mathrm{G})$ are observed in the photoelectron spectra of $\mathrm{Ag}_{6}^{-}$(listed in Table I). The five peaks at lower BE (A-E) display a large similarity to the corresponding peaks $(\mathrm{A}-\mathrm{E})$ in the spectrum of $\mathrm{Cu}_{6}^{-}$. The two spectra of $\mathrm{Ag}_{6}^{-}$ 


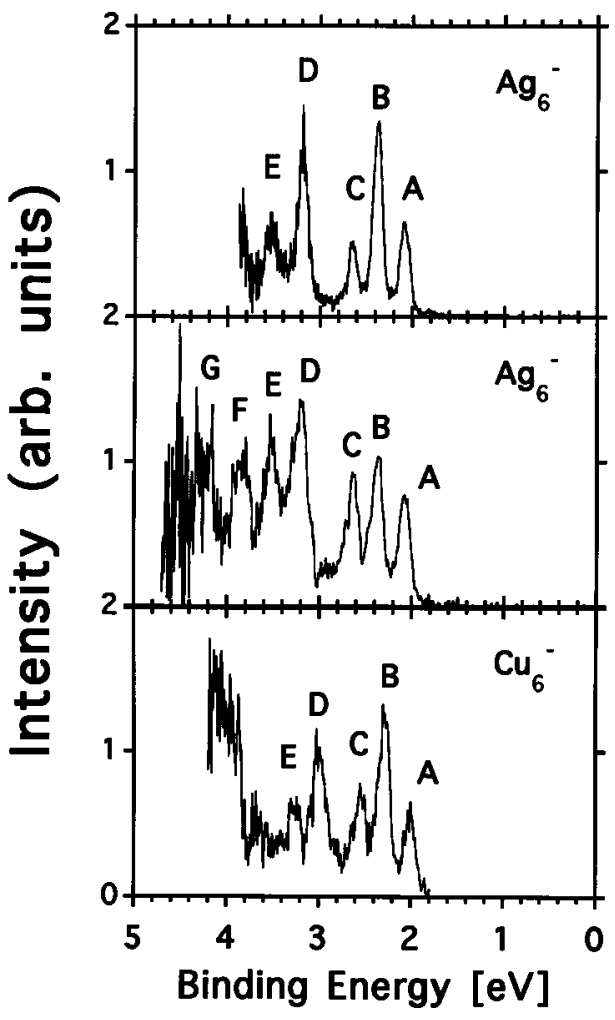

FIG. 8. Comparison of photoelectron spectra of $\mathrm{Ag}_{6}^{-}$recorded at $h \nu=5.0 \mathrm{eV}$ and $h \nu=4.025 \mathrm{eV}$, respectively, with a spectrum of $\mathrm{Cu}_{6}^{-}$recorded at $h \nu=5.0$ $\mathrm{eV}$. An assignment of the marked features (A) $-(\mathrm{G})$ is given in the text.

exhibit pronounced changes in the relative intensities of features $\mathrm{B}$ and $\mathrm{D}$ with photon energy.

According to the calculations, $\mathrm{Ag}_{6}^{-}$has a compact threedimensional shape (tetragonal tripyramid, $C_{2 v}$ symmetry). The electronic ground state of the anion is ${ }^{2} B_{1}$. The calculated energy of the ground state transition $\left({ }^{1} A_{1} \leftarrow{ }^{2} B_{1}\right)$ is in almost perfect agreement with the experiment (see Table I). The BE's of the features corresponding to transitions into the lowest three excited states $\left({ }^{3} B_{1},{ }^{1} B_{1},{ }^{3} A_{2}\right)$ are also in agreement with the spectra. However, the assignment of the features at higher BE's $(E-G)$ is more difficult.

A tentative assignment can be based on the relative intensities of the observed features and the comparison to $\mathrm{Cu}_{6}^{-}$. The seven valence electrons occupy four single particle orbitals. The configuration of the anion ground state is $a_{1}^{2} b_{2}^{2} 2 a_{1}^{2} b_{1}^{1}$. Therefore, the appearance of an uppermost single peak with a relatively low intensity corresponding to photoemission of the unpaired electron is expected. In addition, the photoemission from each fully occupied orbital yields a doublet of two peaks with an intensity ratio of 3/1. From the systematic shift of the lowest $s$-derived orbital with increasing cluster size (feature $\mathrm{F}$ in the spectrum of $\mathrm{Ag}_{5}^{-}$) one expects a $\mathrm{BE}$ of more than $5 \mathrm{eV}$ for the lowest $a_{1}$ orbital of $\mathrm{Ag}_{6}^{-}$. Therefore, direct photoemission should result in at least five peaks within $5 \mathrm{eV}$ BE with estimated relative intensities 1:3:1:3:2 where the two lines of the two doublets should exhibit a similar energy separation (singlet-triplet splitting). This is about the pattern of the five features A-E observed in the spectrum of $\mathrm{Ag}_{6}^{-}$taken at $h \nu=4.0 \mathrm{eV}$ and
$\mathrm{Cu}_{6}^{-}$taken at $h \nu=5.0 \mathrm{eV}$. From these considerations we tentatively assign feature A to photoemission from the $b_{1}$ orbital and the two doublets $\mathrm{B} / \mathrm{C}$ and $\mathrm{D} / \mathrm{E}$ to photoemission from the $2 a_{1}$ and $b_{2}$ orbitals, respectively. At $5 \mathrm{eV}$ photon energy the relative intensities of features $B$ and $D$ are reduced and the intensity ratios between the two multiplet components are no longer in agreement with the expected ratio of $3: 1$. This cannot be explained within the single particle picture and must be due to matrix element effects.

Based on these tentative assignments the spectra can be compared to the calculated data. There are transitions into five states ${ }^{1} A_{1},{ }^{3} B_{1},{ }^{1} B_{1},{ }^{3} A_{2}$, and ${ }^{1} A_{2}$ with the leading configurations $a_{1}^{2} b_{2}^{2} 2 a_{1}^{2}, a_{1}^{2} b_{2}^{2} 2 a_{1}^{1} b_{1}^{1}, a_{1}^{2} b_{2}^{2} 2 a_{1}^{1} b_{1}^{1}, a_{1}^{2} b_{2}^{1} 2 a_{1}^{2} b_{1}^{1}$, and $a_{1}^{2} b_{2}^{1} 2 a_{1}^{2} b_{1}^{1}$, respectively. The five transitions correspond to the emission from the uppermost three occupied orbitals $\left(b_{2}, 2 a_{1}, b_{1}\right)$ of the anion. Therefore, we assign features A, $\mathrm{B}, \mathrm{C}, \mathrm{D}$, and $\mathrm{E}$ to the transitions into these five states in agreement with the tentative assignment. The calculated BE's agree reasonably well with the peak positions (see Table I). The assignment of feature $\mathrm{D}$ to the transition into the ${ }^{3} A_{2}$ state is supported by the photon energy dependence of the peak intensity, which is similar to the other triplet transition (feature $\mathrm{B},{ }^{3} B_{1}$ ). The assignment of peak $\mathrm{E}$ to the transition into the ${ }^{1} A_{2}$ state is based on the assumption of a similar singlet/triplet splitting for the emission from the $2 a_{1}$ and $b_{2}$ orbitals ( 0.3 and $0.34 \mathrm{eV}$, respectively).

From the comparison with the photoelectron spectra of the smaller clusters we expect the position of the doublet corresponding to emission from the $a_{1}$ orbital at a $\mathrm{BE}$ significantly larger than $5 \mathrm{eV}$, which is therefore not observable at present. The two peaks $F$ and $G$ can be assigned to shake-up transitions. Three such transitions have been predicted with BE's at 4.08, 4.12, and $4.51 \mathrm{eV}$ $\left({ }^{3} B_{2},{ }^{3} A_{1},{ }^{1} B_{2} \leftarrow^{2} B_{1}\right)$. The energies differ significantly from the measured peak positions and an unambiguous assignment based on the calculated energies only is not possible at present.

In terms of the shell model the configuration of the $\mathrm{Ag}_{6}^{-}$ ground state is $1 s^{2} 1 p_{1}^{2} 1 p_{2}^{2} 1 p_{3}^{1}$. The $\mathrm{BE}$ of the $1 s^{2}$ orbital is probably shifted beyond $5.0 \mathrm{eV}$ and therefore not visible in our spectra. According to the simple picture of photoemission the appearance of five peaks with the intensity ratio of 1:3:1:3:2 is expected, which can be assigned to photoemission from the three uppermost $1 p$ subshells. Therefore, feature $\mathrm{A}$ is assigned to emission from the $1 p_{3}$ shell and the four features $\mathrm{B} / \mathrm{C}$ and $\mathrm{D} / \mathrm{E}$ are assigned to the doublets corresponding to photoemission from the $1 p_{3}$ and $1 p_{2}$ subshells, respectively. Qualitatively, the assignment is supported by the results of the quantum chemical calculations, since the symmetries of the exact single particle orbitals $a_{1}, b_{2}, 2 a_{1}$, and $b_{1}$ match the ones of the $1 s, 1 p_{1}, 1 p_{2}$, and $1 p_{3}$ subshells, respectively.

$\mathrm{Ag}_{7}^{-}$

Figure 9 displays a comparison of photoelectron spectra of $\mathrm{Ag}_{7}^{-}$recorded at $h \nu=4.0,5.0,6.424 \mathrm{eV}$ and a spectrum of $\mathrm{Cu}_{7}^{-}$recorded at $h \nu=5.0 \mathrm{eV}$. Three peaks (A, B, and C) can be identified in the spectrum of $\mathrm{Ag}_{7}^{-}$at $h \nu=5.0 \mathrm{eV}$ photon energy. The spectrum recorded using $6.424 \mathrm{eV}$ laser radiation 


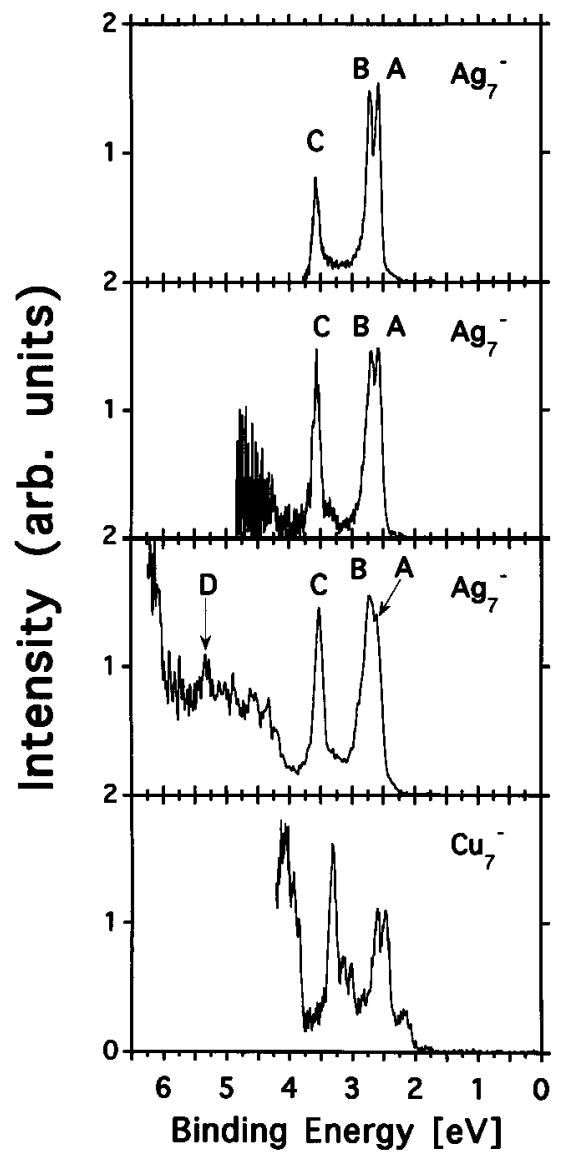

FIG. 9. Comparison of photoelectron spectra of $\mathrm{Ag}_{7}^{-}$recorded at $h \nu=4.025$, 5.0 , and $6.424 \mathrm{eV}$, respectively, with a spectrum of $\mathrm{Cu}_{7}^{-}$recorded at $5.0 \mathrm{eV}$. For a discussion of the marked features (A)-(D) see the text.

exhibits a broad intense feature between 4.0-6.0 eV binding energy.

The calculation of $\mathrm{Ag}_{7}^{-}$assumes two possible geometries, which are close in energy: a capped square bipyramid and a pentagonal bipyramid. However, in both cases the pattern of the calculated transitions does not fit the spectra displayed in Fig. 9. The vertical detachment energy (VDE) of the square bipyramid agrees with the BE of peak A. However, no other transition is predicted up to $4 \mathrm{eV} \mathrm{BE}$ in disagreement with the observation of features $\mathrm{B}$ and $\mathrm{C}$.

A tentative assignment can be based on the comparison with the photoelectron spectrum of $\mathrm{Ag}_{6}^{-}$. The seven electrons of $\mathrm{Ag}_{6}^{-}$occupy four single particle orbitals as do the eight electrons of $\mathrm{Ag}_{7}^{-}$. Since $\mathrm{Ag}_{7}^{-}$is a closed shell species (singlet electronic ground state), only one peak per occupied single particle orbital is expected. The BE's of the four orbitals in $\mathrm{Ag}_{6}^{-}$can be estimated to be about 5.1, 3.4, 2.5, and $2.1 \mathrm{eV}$ (Fig. 8). The BE of the feature corresponding to the lowest orbital $(5.1 \mathrm{eV})$ is estimated from the systematic shift of the corresponding orbitals of $\mathrm{Ag}_{3}^{-}, \mathrm{Ag}_{4}^{-}$, and $\mathrm{Ag}_{5}^{-}$. If a multiplet is observed, the $\mathrm{BE}$ of the corresponding single particle orbital is calculated to be the "center of gravity" of the multiplet. ${ }^{28}$ If it is assumed, that for $\mathrm{Ag}_{7}^{-}$the pattern of these orbitals is basically similar but shifted by $0.3 \mathrm{eV}$ towards higher BE the following pattern is calculated: 5.4, 3.7, 2.8, and $2.4 \mathrm{eV}$. This is about the position of the observed fea- tures $\mathrm{C}-\mathrm{A}(3.60,2.73$, and $2.69 \mathrm{eV})$. At $5.3 \mathrm{eV}$ BE a weak peak (Fig. 9) is superimposed on a broad emission signal. This feature is located at about the BE expected for direct emission from the lowest single particle orbital of $\mathrm{Ag}_{7}^{-}$. However, due to the poor signal to noise ratio, we do not consider this feature in the further discussion. Therefore, we assign the observed features A, B, and C to direct photoemission from the three uppermost occupied single particle orbitals of $\mathrm{Ag}_{7}^{-}$. The slight changes in the energy spacings of the peaks (especially between the nearly degenerate peaks A and B) may be explained by differences in the geometric structures of $\mathrm{Ag}_{6}^{-}$and $\mathrm{Ag}_{7}^{-}$.

The photoelectron spectrum of $\mathrm{Cu}_{7}^{-}$exhibits the same pattern of three intense features within $4 \mathrm{eV} \mathrm{BE}$. The broad peak located between $3.8-4.3 \mathrm{eV}$ is assigned to emission from $3 d$-derived states. Three additional weak features are observed at 2.2, 3.0, and $3.2 \mathrm{eV} \mathrm{BE}$. The two peaks at 3.0 and $3.2 \mathrm{eV} \mathrm{BE}$ are assigned to shake-up processes. ${ }^{18}$ The weak feature at $2.3 \mathrm{eV} \mathrm{BE}$ in the spectrum of $\mathrm{Cu}_{7}^{-}$is assigned to a different, less abundant isomer of this cluster.

The broad feature (4.0-6.0 eV BE) visible in the photoelectron spectrum of $\mathrm{Ag}_{7}^{-}$recorded at $h \nu=6.424 \mathrm{eV}$ results from thermionic emission induced by the absorption of one or several photons. A similar broad feature appears in all spectra of the clusters larger than $\mathrm{Ag}_{7}^{-}$and is also found in the photoelectron spectra of $\mathrm{Ni}_{n}^{-}, \mathrm{W}_{n}^{-}, \mathrm{C}_{n}^{-}$, and $\mathrm{Si}_{n}^{-}$clusters. An analysis of the dependence of this featureless emission signal on the cluster size, the photon flux, the electron affinity and the vibrational temperature of the cluster anions will be published elsewhere. ${ }^{29}$

In terms of the shell model the configuration of $\mathrm{Ag}_{7}^{-}$is $1 s^{2} 1 p_{1}^{2} 1 p_{2}^{2} 1 p_{3}^{2}$. The $1 p$ shell is completely filled and the cluster is expected to be spherical with the $1 p$ subshells to be degenerate. However, according to the above considerations we assign the features $\mathrm{A}, \mathrm{B}, \mathrm{C}$, and $\mathrm{D}$ to direct photoemission from nondegenerate $1 s, 1 p_{1}, 1 p_{2}$, and $1 p_{3}$ subshells in analogy to the discussion of $\mathrm{Ag}_{6}^{-}$. Therefore, $\mathrm{Ag}_{7}^{-}$should deviate from the spherical symmetry, which seems reasonable for a particle with only seven atoms. The pattern of two $1 p$ subshells at lower BE's correlates with a prolate deformation of the cluster. The two geometries calculated using the HF-CI formalism have more oblatelike structures. Since the calculations do not match the photoemission data at all, it is possible, that the two calculated isomers correspond to two local minima within the configuration space, which might not correspond to the global minimum.

$A g_{8}^{-}$

Figure 10 displays a comparison of photoelectron spectra of $\mathrm{Ag}_{8}^{-}$recorded at $h \nu=4.025 \mathrm{eV}$ and $h \nu=5.0 \mathrm{eV}$ with the photoelectron spectrum of $\mathrm{Cu}_{8}^{-}$recorded at $h \nu=5.0 \mathrm{eV}$. Four dominant features $(\mathrm{A}-\mathrm{D})$ can be identified in the spectra of $\mathrm{Ag}_{8}^{-}$, with similar BE's as the corresponding peaks in the spectrum of $\mathrm{Cu}_{8}^{-}$. Additional weak transitions are marked $\mathrm{X}, \mathrm{Y}$, and $\mathrm{Z}$. The relative intensities of $\mathrm{Y}$ and $\mathrm{Z}$ depend strongly on the photon energy. In the spectrum recorded with $h \nu=4.0 \mathrm{eV}$ these two features (X and $\mathrm{Y}$ ) are much weaker and not resolved from peak $\mathrm{C}$.

In $D_{2 d}$ symmetry the electronic ground state of the neutral $\mathrm{Ag}_{8}$ is a closed shell $\left({ }^{1} A_{1}\right)$ configuration with eight va- 


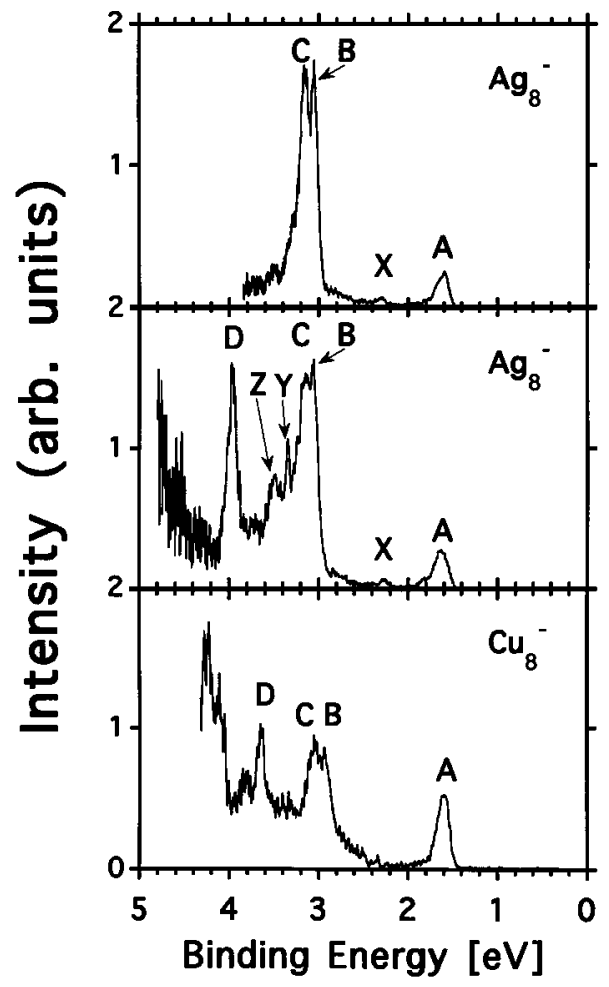

FIG. 10. Comparison of photoelectron spectra of $\mathrm{Ag}_{8}^{-}$recorded at $h \nu$ $=4.025$ and $5.0 \mathrm{eV}$ with the spectrum of $\mathrm{Cu}_{8}^{-}$recorded at $h \nu=5.0 \mathrm{eV}$. The marked features (A)-(D) are discussed in the text.

lence electrons. The additional electron of the anion is only weakly bound resulting in a low $\mathrm{BE}$ of the ground state transition (feature A). Seven transitions have been calculated for $\mathrm{Ag}_{8}^{-}$up to $4.52 \mathrm{eV} \mathrm{BE}$. An assignment of the experimentally observed features to the calculated transitions is based on the observed relative intensities and the measured BE's. Feature $\mathrm{A}$ is assigned to the ground state transition ${ }^{1} A_{1} \leftarrow{ }^{2} A_{1}$. Because of the relatively high and about equal intensities features $\mathrm{B}, \mathrm{C}$, and $\mathrm{D}$ are tentatively assigned to transitions into triplet states corresponding to direct photoemission from one of the occupied single particle orbitals of the anion (configuration: $1 a_{1}^{2} 1 b_{2}^{2} 1 e^{4} 2 a_{1}^{1}$ in $D_{2 d}$ symmetry). Two such transitions are predicted with the final state leading configurations $\left(1 a_{1}^{2} 1 b_{2}^{2} 1 e^{3} 2 a_{1}^{1}\right)$ and $\left(1 a_{1}^{2} 1 b_{2}^{1} 1 e^{4} 2 a_{1}^{1}\right)$ : ${ }^{3} E \leftarrow{ }^{2} A_{1}$ and $2{ }^{3} B_{2} \leftarrow{ }^{2} A_{1}$. The $\mathrm{BE}$ for the transition ${ }^{3} E \leftarrow{ }^{2} A_{1}$ agrees reasonably well with the $\mathrm{BE}$ of feature $\mathrm{B}$, while the $\mathrm{BE}$ of the peak $\mathrm{D}$ is slightly lower $(0.24 \mathrm{eV})$ than the calculated $\mathrm{BE}$ for the $2{ }^{3} B_{2} \leftarrow{ }^{2} A_{1}$ transition (see Table I). The $1 e$ orbital is twofold degenerate. If the symmetry of the anion is slightly perturbed with respect to the $D_{2 d}$ symmetry assumed in the calculations, the twofold degeneracy of the $1 e$ orbital can be lifted and the ${ }^{3} E_{1} \leftarrow{ }^{2} A_{1}$ transition would split into two transitions corresponding to two peaks in the photoelectron spectrum of about equal intensity. Therefore, we assign features $\mathrm{B}$ and $\mathrm{C}$ to direct photoemission from the slightly distorted $1 e$-derived orbitals. If the two features B and C correspond to triplet final states, two weaker peaks are expected at slightly higher BE corresponding to the singlet final states. The calculated singlet-triplet splitting is $0.2 \mathrm{eV}$, which according to the experimental ob- servations on $\mathrm{Ag}_{2}^{-}, \mathrm{Ag}_{4}^{-}$, and $\mathrm{Ag}_{6}^{-}$, is probably overestimated. Therefore, the singlet transition accompanying feature B may be superimposed by feature $\mathrm{C}$ and the singlet peak of feature $\mathrm{C}$ might be hidden in the tail on the high $\mathrm{BE}$ side of this peak. There are two shake-up transitions $\left({ }^{3} B_{2} \leftarrow{ }^{2} A_{1},{ }^{3} A_{1} \leftarrow{ }^{2} A_{1}\right)$ very close in energy to features B and $\mathrm{C}$. An unambiguous assignment of the features $\mathrm{Y}$ and $\mathrm{Z}$ is not possible at present. Feature $\mathrm{X}$ also cannot be assigned.

Feature $\mathrm{D}$ is assigned to the $2{ }^{3} B_{2} \leftarrow{ }^{2} A_{1}$ transition corresponding to direct photoemission from the $1 b_{2}$ orbital. The corresponding singlet transition $2{ }^{1} B_{2} \leftarrow{ }^{2} A_{1}$ is calculated at $0.3 \mathrm{eV}$ higher BE. However, no distinct peak at higher BE beyond feature $\mathrm{D}$ is observed, but peak $\mathrm{D}$ exhibits a slight asymmetry towards higher BE. Therefore, it is possible, that the singlet transition is not resolved from the triplet peak. This would correspond to an upper limit of the singlet-triplet splitting of $0.2 \mathrm{eV}$.

In terms of the shell model the configuration of $\mathrm{Ag}_{8}^{-}$is $1 s^{2} 1 p_{1}^{2} 1 p_{2}^{2} 1 p_{3}^{2} 1 d^{1}$. With a prolate geometry of this cluster anion the $1 p_{2}$ and $1 p_{3}$ orbitals may be degenerate, while the $1 p_{1}$ orbital is located at a higher BE. We assign feature A to the emission from the $1 d$ orbital and $\mathrm{B}, \mathrm{C}$, and $\mathrm{D}$ to emissions from the nearly degenerate $1 p_{2}$ and $1 p_{3}$ orbitals and the $1 p_{1}$ orbital, respectively.

A basic property of the shell model is the similarity of the inner shell structures of different clusters, which in the first place differ by the number of electrons in the outermost shell. This shell principle can be directly probed by comparison of the photoelectron spectra of $\mathrm{Ag}_{7}^{-}$and $\mathrm{Ag}_{8}^{-}$. The patterns of the three dominant features assigned to the fully occupied $1 p$ shell $\left(\mathrm{Ag}_{7}^{-}: \mathrm{A}, \mathrm{B}, \mathrm{C} ; \mathrm{Ag}_{8}^{-}: \mathrm{B}, \mathrm{C}, \mathrm{D}\right)$ is almost identical in both spectra, but shifted towards higher $\mathrm{BE}$ for the larger cluster. Here, this similarity is more pronounced than for the smaller clusters, because with increasing cluster size the energy of the multiplet splitting decreases thus reducing the complexity of the spectra of the clusters with an odd number of electrons. In addition, the change in geometry by adding one electron is less severe.

$\mathrm{Ag}_{9}^{-}$

In Fig. 11 the photoelectron spectra of $\mathrm{Ag}_{9}^{-}$recorded at $h \nu=4.0,5.0$, and $6.424 \mathrm{eV}$ are compared to a photoelectron spectrum of $\mathrm{Cu}_{9}^{-}$recorded at $h \nu=5.0 \mathrm{eV}$. Seven transitions $(A-G)$ can be identified in the photoelectron spectra of $\mathrm{Ag}_{9}^{-}$. For this cluster a significant disagreement with the data of Ho et al. ${ }^{10}$ is observed. In their work only the features A, B, and $\mathrm{C}$ could be observed with a quite different intensity distribution: feature A exhibits the highest relative intensity, while $\mathrm{B}$ and $\mathrm{C}$ have successively lower intensities.

We assign feature $\mathrm{A}$ to a different isomer of $\mathrm{Ag}_{9}^{-}$, which is slightly higher in energy. This explains the higher intensity of this peak in the photoelectron spectrum of Ho et al., because the cooling was less effective in that experiment. The assignment is also supported by the comparison with $\mathrm{Cu}_{9}^{-}$, where the small feature is missing. Three prominent peaks A, $\mathrm{B}$, and $\mathrm{C}$ are observed, which resemble the pattern of the three corresponding features $\mathrm{B}, \mathrm{C}$, and $\mathrm{D}$ in the spectra of $\mathrm{Ag}_{9}^{-}$. The difference in stability of the two corresponding isomers of $\mathrm{Cu}_{9}^{-}$might be larger than in the case of $\mathrm{Ag}$ and therefore only one isomer is observed. 


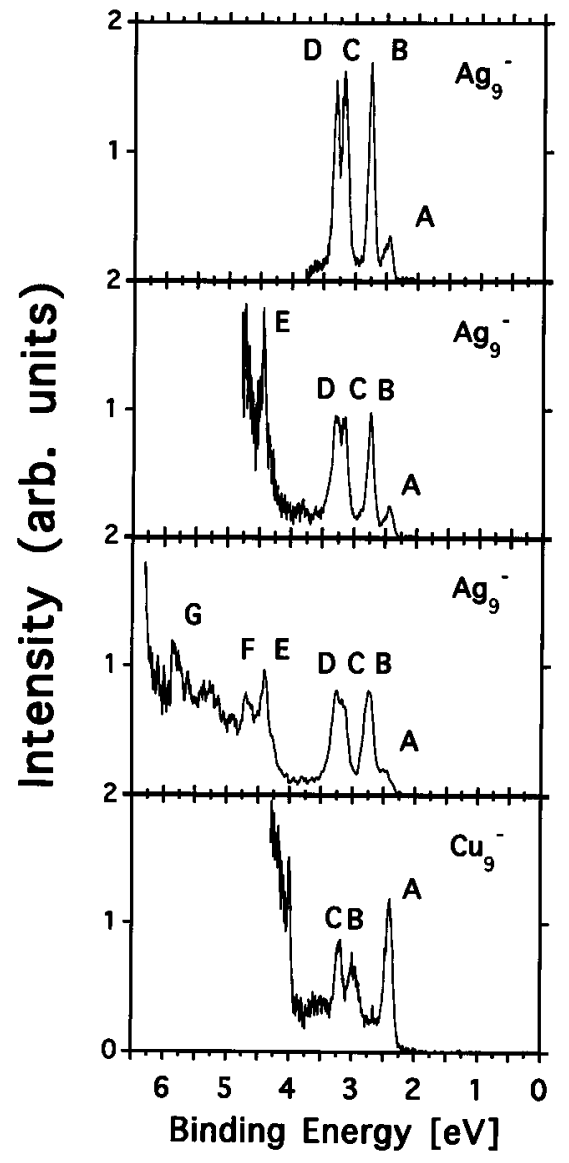

FIG. 11. Comparison of photoelectron spectra of $\mathrm{Ag}_{9}^{-}$recorded at $h \nu$ $=4.025,5.0$, and $6.424 \mathrm{eV}$, respectively, with a spectrum of $\mathrm{Cu}_{9}^{-}$recorded at $h \nu=5.0 \mathrm{eV}$. For a discussion of the marked features $(\mathrm{A})-(\mathrm{G})$ see the text.

At present it is not possible to assign the observed features to the results of the HF-CI calculations. The BE's of the features A, C, and D $(2.45,3.19,3.33 \mathrm{eV}$, respectively) agree within $0.2 \mathrm{eV}$ with the calculated energies of the transitions from the anion ground state ${ }^{1} A^{\prime}$ into the neutral ground state ${ }^{2} A^{\prime}(2.34 \mathrm{eV})$ and the first and second excited states ${ }^{2} A^{\prime \prime}$ $(3.13 \mathrm{eV})$ and ${ }^{2} A^{\prime}(3.45 \mathrm{eV})$ assuming a $C_{s}$ symmetry of the anion. However, this possible assignment disagrees with the above assignment of feature $\mathrm{A}$ to a different isomer. In addition, feature B cannot be assigned to any transition for this geometry. There are also calculations of a different isomer of $\mathrm{Ag}_{9}^{-}$with a $C_{2 v}$ symmetry. However, for both calculated isomers and also if a mixture of the two isomers is assumed, it is not possible to assign the measured photoelectron data.

Based on the shell model a tentative assignment of the various features can be made (see Table I). The configuration of $\mathrm{Ag}_{9}^{-}$is $1 s^{2} 1 p_{1}^{2} 1 p_{2}^{2} 1 p_{3}^{2} 1 d^{2}$. This ten-electron cluster should assume a prolate shape similar to $\mathrm{Ag}_{8}^{-}$. Therefore, a certain similarity of the photoelectron spectrum of $\mathrm{Ag}_{8}^{-}$with the one of $\mathrm{Ag}_{9}^{-}$is expected. A corresponding similarity has been found ${ }^{18}$ for the spectra of $\mathrm{Cu}_{8}^{-}$and $\mathrm{Cu}_{9}^{-}$as well, where only the ground state transition and peak $\mathrm{D}$ are shifted towards lower $\mathrm{BE}$ for $\mathrm{Cu}_{9}^{-}$. Based on these considerations, we assign feature $\mathrm{B}$ to the $1 d$ subshell and features $\mathrm{C}, \mathrm{D}$, and $\mathrm{E}$ to the $1 p_{3}, 1 p_{2}$, and $1 p_{1}$ subshells, respectively. Feature $\mathrm{F}$ cannot be assigned based on the shell model and is probably due to a shake-up transition. The $1 s$ shell is expected to be at a BE larger than $5.5 \mathrm{eV}$. Therefore, feature $\mathrm{G}$ is a possible candidate for emission from the $1 s$ shell.

$A g_{10}^{-}-A g_{21}^{-}$

Since calculations are not available for the comparison with the spectra of the larger clusters, only a tentative assignment based on the predictions of the shell model is possible. These assignments are analogous to the discussion of the data of the $\mathrm{Cu}_{n}^{-}$clusters. ${ }^{18}$ According to the above comparison of HF-CI calculations with the $\mathrm{Ag}_{n}^{-}$data, the earlier tentative assignment of the $\mathrm{Cu}_{n}^{-}$with $n=2-9$ based on the shell model was basically correct. We conclude, that qualitatively this approach is likely correct for the larger clusters, too.

Figures 12(a)-12(d) show the photoelectron spectra of $\mathrm{Ag}_{n}^{-}$with $n=10-21(n=10-18$ for $h \nu=4.66 \mathrm{eV})$ taken at $h \nu=4.025,4.66,5.0$, and $6.424 \mathrm{eV}$. The data are recorded at a higher energy resolution than the corresponding data ${ }^{18}$ on $\mathrm{Cu}_{n}^{-}$and display more spectral fine structure. Only the dominant structures are assigned to electronic shells. We assume, that the orbitals are nondegenerate single particle orbitals corresponding to a nonspherical symmetry of the clusters (except for $\mathrm{Ag}_{17}^{-}$). The electrons are successively filled into the lowest orbitals according to the shell structure. Thus the 22 electrons of $\mathrm{Ag}_{21}^{-}$lead to the configuration $1 s^{2} 1 p_{1}^{2} 1 p_{2}^{2} 1 p_{3}^{2} 1 d_{1}^{2} 1 d_{2}^{2} 1 d_{3}^{2} 1 d_{4}^{2} 1 d_{5}^{2} 2 s^{2} 1 f_{1}^{2}$. It is assumed, that the $\mathrm{BE}$ of each single particle orbital increases monotonically with increasing size of the cluster. The BE's of all identified features and the shell assignments are listed in Table I.

$\mathrm{Ag}_{10}^{-}$

The five dominant peaks (A, B, C, E, and F) observed in the spectra of $\mathrm{Ag}_{10}^{-}$can be assigned to photoemission from electronic shells (Table I). The weak feature D is tentatively assigned to a shake-up process. The peaks $\mathrm{C}$ and $\mathrm{E}$ exhibit a finestructure, which cannot be explained at present. A similar pattern of four dominant peaks $(\mathrm{A}, \mathrm{B}, \mathrm{C}$, and $\mathrm{E})$ is also observed in the spectrum ${ }^{18}$ of $\mathrm{Cu}_{10}^{-}$.

$A g_{11}^{-}$

The five pronounced features $(\mathrm{B}, \mathrm{C}, \mathrm{D}, \mathrm{E}$, and $\mathrm{H})$ are assigned to electronic shells. In the photoelectron spectra recorded at $h \nu=4.025 \mathrm{eV}$ and $h \nu=5.0 \mathrm{eV}$ an additional weak peak (labeled A) is observed at very low BE. The photoelectron spectrum ${ }^{18}$ of $\mathrm{Cu}_{11}^{-}$exhibits a similar pattern of four features at about the BE's of the peaks B, C, D, and E but not the peak at very low BE. Therefore, feature $\mathrm{A}$ is not assigned to a photoemission peak of the dominant isomer of $\mathrm{Ag}_{11}^{-}$. Some other photoelectron spectra of the $\mathrm{Ag}_{n}^{-}$clusters (e.g., $\left.\mathrm{Ag}_{9}^{-}, \mathrm{Ag}_{13}^{-}\right)$exhibit similar weak features at low BE's. As discussed for $\mathrm{Ag}_{9}^{-}$we assign these features to photoemission from isomers other than the most stable one. In Table I these features are labeled with "Isomer."

The features $\mathrm{F}$ and $\mathrm{G}$ are not observed in the corresponding spectrum of $\mathrm{Cu}_{11}^{-}$and cannot be explained within the shell model. We tentatively assign these features to shake-up processes.

$\mathrm{Ag}_{12}^{-}$

Again the five most intense features (A, B, C, D, and F) are assigned to electronic shells (Table I). The weak feature 

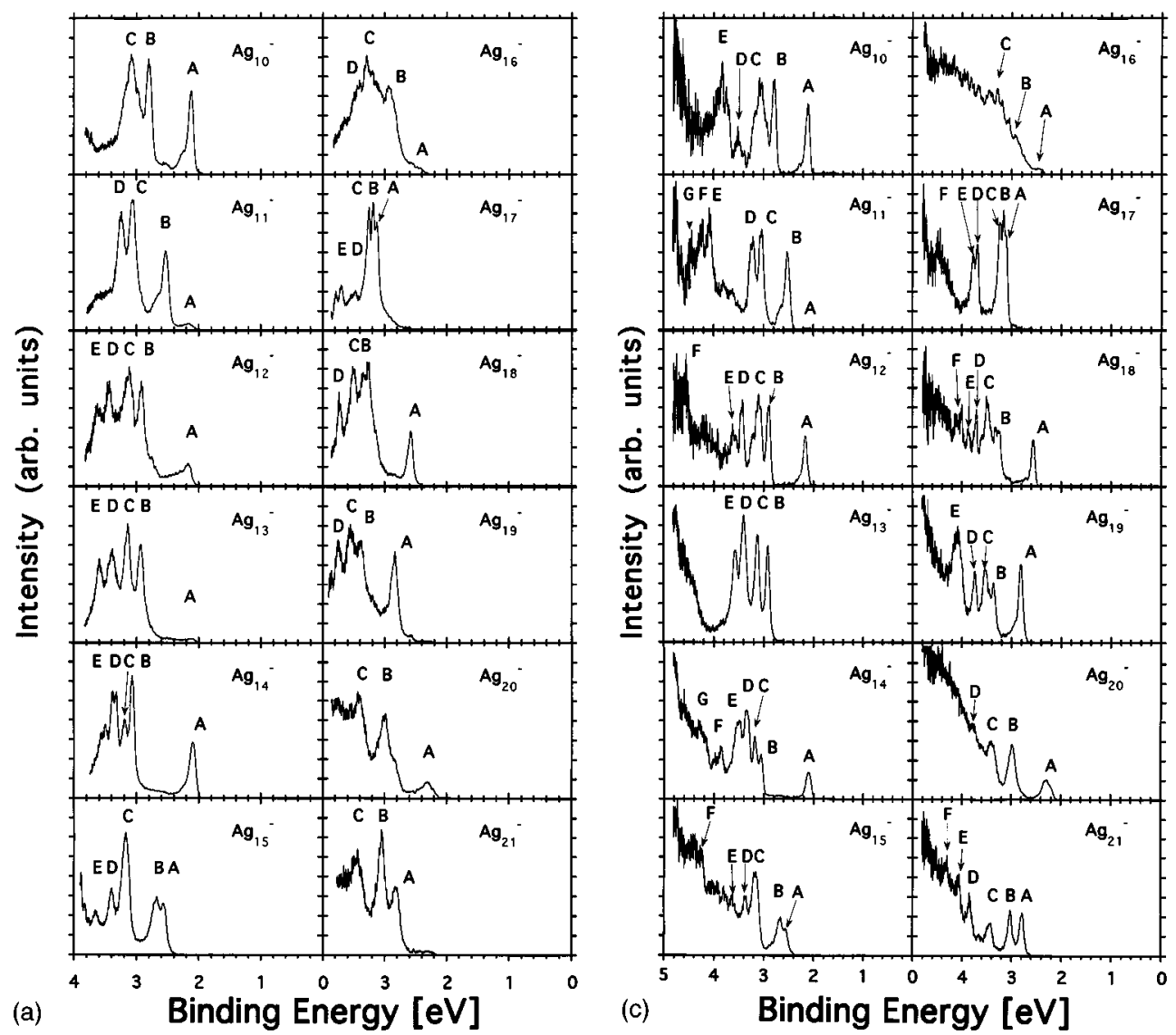

(c)
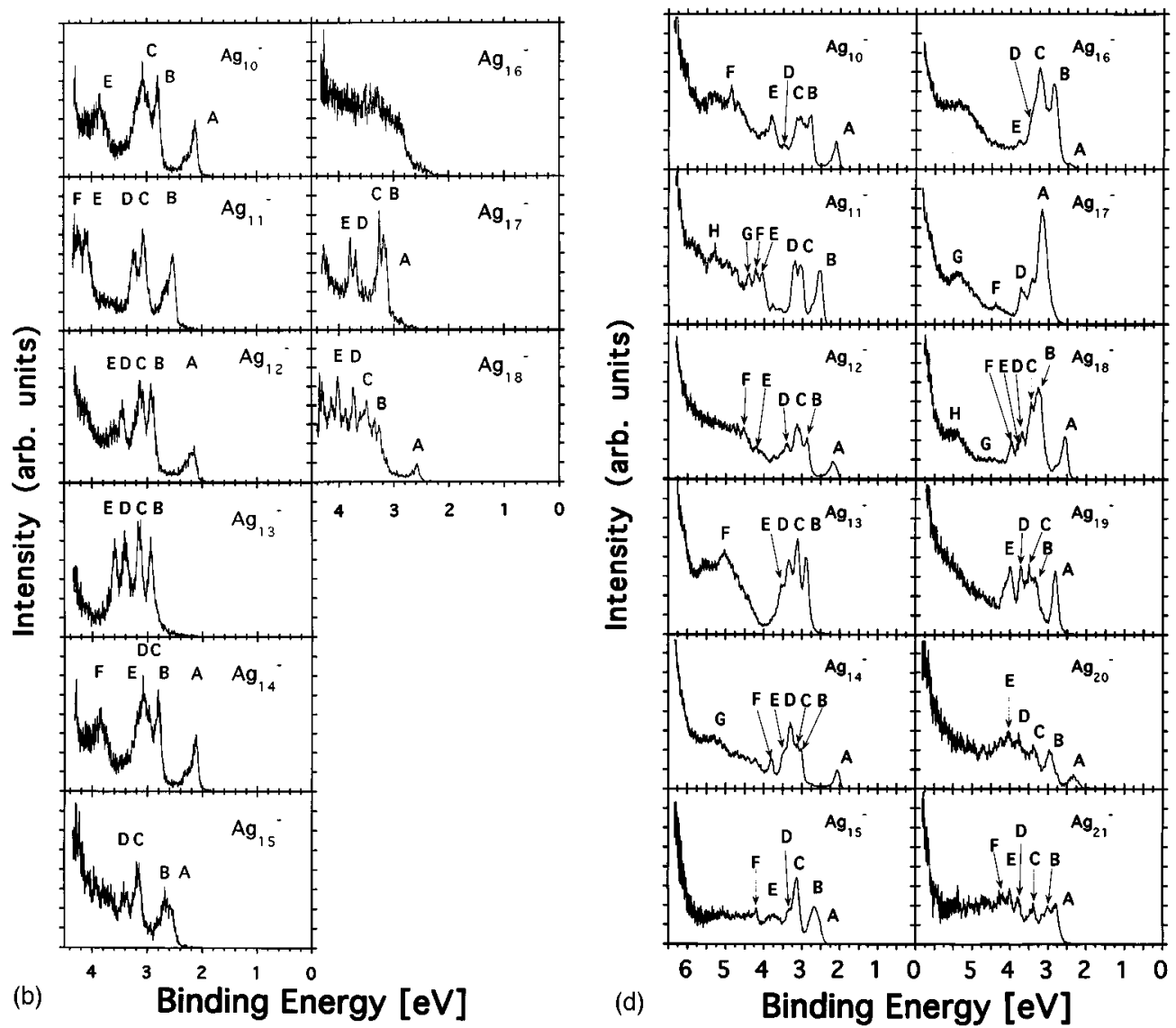

FIG. 12. Photoelectron spectra of $\mathrm{Ag}_{n}^{-}$with $n=10-21$ taken at $h \nu=4.0 \mathrm{eV}$ (a), $4.66 \mathrm{eV}$ (b), $5.0 \mathrm{eV}$ (c), and $6.424 \mathrm{eV}$ (d). A discussion of the various marked features is given in the text. 
$\mathrm{E}$ is assigned to a shake-up process.

$\mathrm{Ag}_{13}^{-}$

As for the previous clusters the five dominant features (B, C, D, E, and F) are assigned to electronic shells (Table I). Again a weak photoemission signal at very low BE (feature A) is observed, which is assigned to photoemission from another isomer.

$\operatorname{Ag}_{14}^{-}$

Based on the comparison with the spectrum of $\mathrm{Cu}_{14}^{-}$the dominant features A, B, C, D, E, F, and G are assigned to electronic shells (Table I). Feature $\mathrm{C}$ is assigned to a shake-up process.

$\operatorname{Ag}_{15}^{-}$

Based on the comparison with the spectrum of $\mathrm{Cu}_{15}^{-}$the dominant features A, C, D, E, and F are assigned to electronic shells (Table I). Close to the ground state transition A a second peak $\mathrm{B}$ is observed, while in the spectrum of $\mathrm{Cu}_{15}^{-}$ only one feature is observed in this $\mathrm{BE}$ range. In addition, according to the shell model only one feature is expected at this BE. Peak $\mathrm{A}$ in the spectrum of $\mathrm{Ag}_{8}^{-}$exhibits a similar finestructure. A second feature seems to be located very close to peak A (Fig. 8) at slightly higher BE. Since no electronic effect can be found explaining these double features, we tentatively assign the peaks to photoemission from other isomers. In the case of $\mathrm{Ag}_{15}^{-}$it cannot be decided, which feature (A or B) has to be assigned to the isomer with the lower ground state energy. We tentatively assign feature A to the ground state isomer.

$\mathrm{Ag}_{\overline{16}}^{-}$

The spectrum of $\mathrm{Ag}_{16}^{-}$recorded at $h \nu=5.0 \mathrm{eV}$ displays a relatively smooth increasing photoemission signal with only small peaks superimposed. However, in spectra taken at $h \nu$ $=4.0 \mathrm{eV}$ and at $h \nu=6.424 \mathrm{eV}$ five distinct features (A-D) can be identified. The five features are tentatively assigned to photoemission from electronic shells (Table I).

$A g_{17}^{-}$

The spectrum of $\mathrm{Ag}_{17}^{-}$displays a prominent peak at 3.2 $\mathrm{eV} \mathrm{BE}$ with a finestructure corresponding to at least three transitions (A, B, and C). The spectrum recorded at $h \nu=5.0$ $\mathrm{eV}$ exhibits a double peak (D and $\mathrm{E}$ ) at about $0.5 \mathrm{eV}$ higher $\mathrm{BE}$, which is much weaker in intensity in the spectra recorded at the other two photon energies. The spectrum recorded at $h \nu=4.66 \mathrm{eV}$ is very similar to the one recorded at $h \nu=5.0 \mathrm{eV}$. The corresponding spectrum of $\mathrm{Cu}_{17}^{-}$recorded at $h \nu=5.0 \mathrm{eV}$ displays only one intense feature at $3.2 \mathrm{eV} \mathrm{BE}$.

The peaks marked $\mathrm{D}$ and $\mathrm{E}$ in the spectra of $\mathrm{Ag}_{17}^{-}$can be assigned to either shake-up processes or to direct photoemission. The strong dependence of the relative intensity on the photon energy indicates shake-up processes. The features assigned to direct photoemission in the photoelectron spectra do not exhibit a comparably strong dependence on the photon energy except for feature $\mathrm{A}$ in the spectrum of $\mathrm{Ag}_{3}^{-}$. However, in the case of the trimer this strong dependence can be explained by the significant difference in the symmetries of the involved electronic states. For $\mathrm{Ag}_{17}^{-}$, these symmetries are expected to be similar for the features A-D.

Another support of the assignment to shake-up processes is the lack of a corresponding feature in the spectrum of $\mathrm{Cu}_{17}^{-}$. Most of the cluster spectra exhibit a large similarity for
$\mathrm{Cu}_{n}^{-}$and $\mathrm{Ag}_{n}^{-}$within the binding energy range studied. A significant difference between a $\mathrm{Cu}$ and a corresponding $\mathrm{Ag}$ spectrum concerning a shake-up feature can be explained by the strong dependence of the relative intensities of these features on details of the involved wave functions.

In terms of the shell model the lowest unoccupied orbital of $\mathrm{Ag}_{17}^{-}$(18 electrons) is the $2 s$ shell. The energy gap between the $1 d$ and the $2 s$ shells can be estimated from the difference in $\mathrm{BE}$ between features $\mathrm{A}$ and $\mathrm{B}$ in the spectrum of $\mathrm{Ag}_{18}^{-}$(see below). Feature $\mathrm{A}$ is assigned to photoemission from the $2 s$ shell and feature $\mathrm{B}$ to the emission from the uppermost occupied $1 d$ subshell. The separation is $0.73 \mathrm{eV}$. This is expected to be comparable to the energy necessary to promote a $1 d$ electron in $\mathrm{Ag}_{17}$ into the $2 s$ orbital. The separation in energy between the dominant group of features in the spectrum of $\mathrm{Ag}_{17}^{-}(\mathrm{A}, \mathrm{B}$, and $\mathrm{C})$ and the supposed shake-up features ( $\mathrm{D}$ and $\mathrm{E}$ ) is about $0.55 \mathrm{eV}$. Therefore, the features $\mathrm{D}$ and $\mathrm{E}$ can be assigned to $2 s \leftarrow 1 d$ shake-up processes accompanying the photoemission from the $1 d$ shell.

The peaks $\mathrm{A}, \mathrm{B}$, and $\mathrm{C}$ are assigned to the $1 d$ emission. The narrow peak corresponding to the emission from the five $1 d$ subshells indicates a significant degree of degeneracy corresponding to a high, almost spherical symmetry of the cluster.

$\mathrm{Ag}_{18}^{-}$

The six identified features $\mathrm{A}-\mathrm{F}$ are assigned to photoemission from electronic shells. Neutral $\mathrm{Ag}_{18}$ is a closed shell species with 18 electrons. The additional electron of the anion occupies the lowest unoccupied molecular orbital of the neutral, which corresponds to the $2 s$ electronic shell. Therefore, the energy separation between feature A (emission from the $2 s$ shell) and feature B (emission from the uppermost $1 d$ subshell) corresponds to the HOMO-LUMO gap transition energy, which is synonymous for the bandgap of the cluster.

$\mathrm{Ag}_{\overline{19}}^{-}$

Again, the five dominant features $(\mathrm{A}-\mathrm{E})$ are assigned to emission from electronic shells. The configuration of $\mathrm{Ag}_{19}^{-}$is $\left(1 s^{2} 1 p^{6}\right) 1 d_{1}^{2} 1 d_{2}^{2} 1 d_{3}^{2} 1 d_{4}^{2} 1 d_{5}^{2} 2 s^{2}$. If the cluster is nonspherical, six features are expected within the studied range of BE's. However, only five distinct peaks are observed.

$\mathrm{Ag}_{20}^{-}$and $\mathrm{Ag}_{21}^{-}$

In contrast to the spectra of $\mathrm{Ag}_{17}^{-}-\mathrm{Ag}_{19}^{-}$the spectra of $\mathrm{Ag}_{20}^{-}$and $\mathrm{Ag}_{21}^{-}$recorded at $h \nu=6.424 \mathrm{eV}$ no longer exhibit a group of lines well separated from the emission signal at higher BE. At low BE several peaks can be identified. At higher BE the features seem to merge into the smooth emission signal. Therefore, the identification and assignment of the features at higher BE's are rather uncertain. A tentative assignment of the first pronounced peaks of $\mathrm{Ag}_{20}^{-}$and $\mathrm{Ag}_{21}^{-}$is included in Table I.

\section{COMPARISON OF THE SHELL MODEL WITH THE QUANTUMCHEMICAL APPROACH}

The results of the quantum chemical calculations on $\mathrm{Ag}_{n}^{-}$with $n=2,3,4,5,6$, and 8 can be used to quantitatively explain most of the important structures in the measured photoelectron spectra. The calculated peak positions deviate from the experimental values by less than $0.3 \mathrm{eV}$. The spectra 


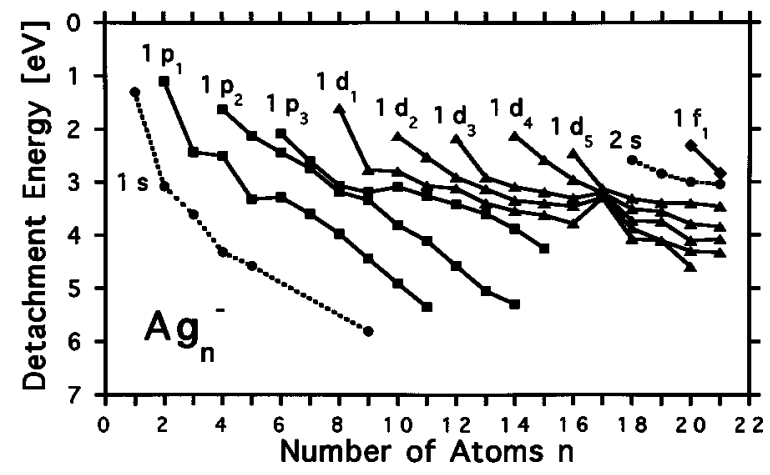

FIG. 13. The BE's of the single particle orbitals extracted from the spectra for each $\mathrm{Ag}_{n}^{-}$cluster.

of $\mathrm{Ag}_{7}^{-}$and $\mathrm{Ag}_{9}^{-}$, however, do not agree with the calculations. The reason is possibly a different geometric structure of the anion. The following information can be gained from the comparison of quantum chemical theory and experiment:

(1) The geometric structure of the anion ground state.

(2) The electronic states of the neutral cluster in the equilibrium geometry of the anion.

However, a qualitative understanding of trends and patterns developing with increasing cluster size is difficult to gain from the quantum chemical description since each and every cluster is treated as an individual molecule.

On the other hand, the dominant features in the photoemission spectra can also be assigned to direct photoemission from occupied single particle orbitals. This assignment can only be qualitative, because the binding energy of a single particle orbital in the anion and the measured position of a feature in the spectrum differ by the relaxation energy since the photoelectron spectrum represents the amount of energy needed to remove an electron from the occupied single particle orbitals in the cluster. Additionally, the spectrum has to be corrected for shake-up processes and multiplet splittings.

The comparison of the assignment to emission from occupied orbitals with the assignment to calculated transitions based on quantum chemical calculations is a test for the validity of the simple interpretation. In all cases, where the quantum chemical calculations predicted states in agreement with the experimental observations the simple interpretation turned out to be qualitatively correct also. This clearly supports the earlier assignments ${ }^{18}$ of the data on $\mathrm{Cu}_{n}^{-}$.

The correspondence between the shell model assignments and the quantum chemical states not only refers to the energies, splittings, and numbers of states, but also to the symmetries. As far as the transitions correspond predominantly to the removal of a single electron, the symmetry of that particular single particle wave function is identical with the symmetry of the assigned subshell. In this framework the position of a direct photoemission feature corresponds to the vertical detachment energies (VDE's) of the single particle orbitals. Figure 13 shows the resulting VDE's of these single particle orbitals extracted from the spectra for each $\mathrm{Ag}_{n}^{-}$ cluster. If a multiplet is observed, the center of gravity of the multiplet peaks has been plotted. Immediately this picture relays the following:

(1) The " $4 s$ bandwidth" and the number of occupied single particle orbitals increases monotonically with $n$.

(2) The orbitals are largely nondegenerate (except for $\mathrm{Ag}_{17}^{-}$).

The identified orbitals can be compared to the predictions of the shell model. According to the above discussion every single particle orbital is assigned to an electronic shell (see Table I) and marked in Fig. 13. Orbitals assigned to the same shell or subshell are connected.

With the shell model assignments significant information about the electronic and geometric structures of the clusters can be extracted from Fig. 13. An oblate deformation results in an enhanced binding energy of two of the $p$ orbitals, while a prolate deformation results in an enhanced binding energy of just one $p$ orbital. A pattern of two $p$ orbitals with a low VDE and one with a higher VDE corresponds to a prolate shape. According to the observed splittings of the $1 p$ subshells the clusters $\mathrm{Ag}_{6}^{-}-\mathrm{Ag}_{9}^{-}$have a prolate shape, while the larger clusters up to $n=14$ exhibit an oblate deformation.

$\mathrm{Ag}_{17}^{-}$seems to be the smallest cluster with an almost spherical symmetry according to the degeneracy of the $1 d$ subshells. Although between $\mathrm{Ag}_{17}^{-}$and $\mathrm{Ag}_{19}^{-}$the $2 s$ shell is filled these clusters are again nonspherical. This is surprising, since an additional electron occupying an $s$ orbital is not expected to induce a deformation. It is possible, that geometric packing effects also influence the shape of the clusters. Nineteen atoms correspond to a stable geometric configuration proposed, e.g., $\mathrm{Xe}_{19}$ which is a capped icosahedron with a prolate shape. ${ }^{30}$ It is possible, that this packing effect disturbs the spherical symmetry given by the electrons. Beyond $\mathrm{Ag}_{19}^{-}$the $1 d$ orbitals split further indicating an even lower symmetry corresponding to the filling of the $1 f$ shell. These observations are basically identical to the pattern observed for $\mathrm{Cu}_{n}^{-}$.

The assignment of the features observed in the photoelectron spectra reflecting the occupied single particle orbitals and the interpretation in terms of the electronic shell model yields a qualitative picture of the development of the electronic structure of clusters of simple metals. For the interpretation of the photoelectron data both models, the quantum chemical approach and the shell model, are required: The first one to gain a sound and quantitative description of the data and the latter one to extract an understanding of the development of the electronic structure of these metal clusters with increasing size. We found no fundamental disagreement between the two interpretations.

However, there is still no experimental proof for the basic assumption of the electronic shell model of a defined angular momentum. This would require the direct determination of the angular momentum of the electrons.

\section{CONCLUSION}

Photoelectron spectra of $\mathrm{Ag}_{n}^{-}$clusters recorded at several different photon energies are compared to similar data on $\mathrm{Cu}_{n}^{-}$clusters and to the results of Hartree-Fock configuration interaction calculations. The calculated energetic position of the transitions from the anionic electronic ground 
states to the neutral ground and excited states are within 0.3 $\mathrm{eV}$ in agreement with the experimental data for $\mathrm{Ag}_{n}^{-}$with $n=2,3,4,5,6$, and 8 . This comparison yields the assignment of almost all features observed in the spectra to the ground and excited electronic states of the neutral cluster in the geometry of the anion.

Based on the calculations, the features in the spectra are classified into direct photoemission from single particle orbitals of the anions and shake-up features. The BE's of the occupied single particle orbitals of the ground state anions are estimated including the effect of multiplet splitting. The assignment to shake-up processes and multiplets is supported by the comparison with the spectra of $\mathrm{Cu}_{n}^{-}$clusters and the photon energy dependence of the relative intensities of the transitions. Finally, this evaluation of the spectra yields a qualitative picture of the occupied single particle orbitals in the cluster anions.

This pattern resulting from the comparison of the data and the calculations is compared to the predictions of the electronic shell model. Each single particle orbital is tentatively assigned to an electronic subshell. The assignments are in agreement with the predicted symmetries of the single particle orbitals calculated using the Hartree-Fock scheme. This evaluation is extended to larger clusters based on the electronic shell model only.

From the above analysis a pattern of the development of the electronic shells of $\mathrm{Ag}_{n}^{-}$clusters is extracted. Up to $n=16$ the clusters are nonspherical resulting in a splitting of the electronic shells into subshells. The clusters with $n=6,7$, and 8 atoms seem to have a prolate geometry. A pronounced shell closing is observed for $\mathrm{Ag}_{17}^{-}$(18 electrons). The levels assigned to the $1 d$ subshells are almost degenerate. We consider this cluster as the smallest one with an almost spherical symmetry. For the larger clusters with $n_{e}=19,20,21$, and 22 electrons the $1 d$ levels are split into a broad band again. The pattern of the electronic shells of $\mathrm{Ag}_{n}^{-}$clusters is almost identical to the one found in $\mathrm{Cu}_{n}^{-}$clusters published in an earlier work ${ }^{18}$ based on a tentative assignment only.

\section{ACKNOWLEDGMENTS}

We thank V. Bonacic-Koutecky for lively and encouraging discussions and the sending of additional results of calculations. H. Pfeifer and J. Lauer are acknowledged for their technical support.
${ }^{1}$ W. de Heer, Rev. Mod. Phys. 65, 611 (1993), and references therein.

${ }^{2}$ D. G. Leopold, J. H. Ho, and W. C. Lineberger, J. Chem. Phys. 86, 1715 (1987)

${ }^{3}$ O. Cheshnovsky, S. H. Yang, P. L. Pettiette, M. J. Craycraft, and R. E. Smalley, Rev. Sci. Instrum. 58, 2131 (1987).

${ }^{4}$ G. Ganteför, K. H. Meiwes-Broer, and H. O. Lutz, Phys. Rev. A 37, 2716 (1988).

${ }^{5}$ D. W. Arnold, S. E. Bradforth, T. N. Kitsopoulos, and D. M. Neumark, J. Chem. Phys. 95, 8753 (1991)

${ }^{6}$ K. M. McHugh, J. G. Eaton, G. H. Lee, H. W. Sarkas, L. H. Kidder, J. T. Snodgrass, M. R. Manaa, and K. H. Bowen, J. Chem. Phys. 91, 3792 (1989).

${ }^{7}$ D. Bahatt, O. Cheshnovsky, and U. Even, Z. Phys. Chem. 184, 253 (1994).

${ }^{8}$ A. Nakajima, T. Taguwa, and K. Kaya, Chem. Phys. Lett. 221, 436 (1994).

${ }^{9}$ C. Y. Cha, G. Ganteför, and W. Eberhardt, Rev. Sci. Instrum. 63, 5661 (1992).

${ }^{10}$ J. Ho, K. M. Ervin, and W. C. Lineberger, J. Chem. Phys. 93, 6987 (1990).

${ }^{11}$ O. Cheshnovsky, K. J. Taylor, J. Conceicao, and R. E. Smalley, Phys. Rev. Lett. 64, 1785 (1990).

${ }^{12}$ K. J. Taylor, C. L. Pettiette-Hall, O. Cheshnovsky, and R. E. Smalley, J. Chem. Phys. 96, 3319 (1992).

${ }^{13}$ G. Ganteför, M. Gausa, K. H. Meiwes-Broer, and H. O. Lutz, Faraday Discuss. Chem. Soc. 86, 1 (1988)

${ }^{14}$ V. E. Bondybey and J. E. English, J. Chem. Phys. 74, 6978 (1981); T. G. Dietz, M. A. Duncan, D. E. Powers, and R. E. Smalley, J. Chem. Phys. 74, 6511 (1981).

${ }^{15}$ G. Ganteför, H. R. Siekmann, H. O. Lutz, and K.-H. Meiwes-Broer, Chem. Phys. Lett. 165, 293 (1990).

${ }^{16}$ G. F. Gantefor, D. M. Cox, and A. Kaldor, J. Chem. Phys. 93, 8395 (1990); 94, 854 (1991).

${ }^{17}$ H. Handschuh, G. Ganteför, P. S. Bechthold, and W. Eberhardt, Rev. Sci. Instrum. (submitted, 1995).

${ }^{18}$ C.-Y. Cha, G. Ganteför, and W. Eberhardt, J. Chem. Phys. 99, 6308 (1993)

${ }^{19}$ V. L. Moruzzi, J. F. Janak, and A. R. Williams, Calculated Electronic Properties of Metals (Pergamon, New York, 1981).

${ }^{20}$ P. Kruit and F. H. Read, J. Phys. E 16, 313 (1983).

${ }^{21}$ H. Hotop and W. C. Lineberger, J. Phys. Chem. Ref. Data 14, 731 (1985).

${ }^{22}$ R. O. Jones, J. Chem. Phys. 99, 1194 (1993).

${ }^{23}$ V. Bonacic-Koutecky, P. Fantucci, and J. Koutecky, J. Chem. Phys. 91, 3794 (1989)

${ }^{24}$ V. Bonacic-Koutecky, L. Cespiva, P. Fantucci, J. Pittner, and J. Koutecky, J. Chem. Phys. 100, 490 (1994).

${ }^{25}$ C.-Y. Cha, G. Ganteför, and W. Eberhardt, J. Chem. Phys. 100, 995 (1994).

${ }^{26}$ H. Handschuh, G. Ganteför, P. S. Bechthold, and W. Eberhardt, J. Chem. Phys. 100, 7093 (1994).

${ }^{27}$ C. F. Moore, Natl. Bur. Stand., Ref. Data Ser. 35 (Natl. Bur. Stand., Washington, D.C., 1971).

${ }^{28}$ J. Berkowitz, Photoabsorption, Photoionization and Photoelectron Spectroscopy (Academic, New York, 1979).

${ }^{29}$ H. Weidele, D. Kreisle, E. Recknagel, G. Schulze Icking-Konert, H. Handschuh, G. Ganteför, P. S. Bechthold, and W. Eberhardt, Chem. Phys. Lett. (submitted, 1995).

${ }^{30}$ O. Echt, K. Sattler, and E. Recknagel, Phys. Rev. Lett. 47, 1121 (1981). 